\title{
ASYMPTOTICS OF THE FAST DIFFUSION EQUATION VIA ENTROPY ESTIMATES
}

\author{
ADRIEN BLANCHET, MATTEO BONFORTE, JEAN DOLBEAULT, GABRIELE GRILLO, AND JUAN \\ LUIS VÁZQUEZ
}

\begin{abstract}
We consider non-negative solutions of the fast diffusion equation $u_{t}=\Delta u^{m}$ with $m \in(0,1)$, in the Euclidean space $\mathbb{R}^{d}, d \geq 3$, and study the asymptotic behavior of a natural class of solutions, in the limit corresponding to $t \rightarrow \infty$ for $m \geq m_{c}=(d-2) / d$, or as $t$ approaches the extinction time when $m<m_{c}$. For a class of initial data we prove that the solution converges with a polynomial rate to a self-similar solution, for $t$ large enough if $m \geq m_{c}$, or close enough to the extinction time if $m<m_{c}$. Such results are new in the range $m \leq m_{c}$ where previous approaches fail. In the range $m_{c}<m<1$ we improve on known results.
\end{abstract}

\section{INTRODUCTION}

We study the Cauchy problem for the fast diffusion equation posed in the whole Euclidean space, that is, we consider the solutions $u(\tau, y)$ of

$$
\left\{\begin{array}{l}
\partial_{\tau} u=\Delta u^{m} \\
u(0, \cdot)=u_{0},
\end{array}\right.
$$

where $m \in(0,1)$ (which means fast diffusion) and $(\tau, y) \in(0, T) \times \mathbb{R}^{d}$ for some $T>0$. We consider non-negative initial data and solutions. Existence and uniqueness of weak solutions of this problem with initial data in $\mathrm{L}_{\mathrm{loc}}^{1}\left(\mathbb{R}^{d}\right)$ was first proved by M.A. Herrero and M. Pierre in [30]. In the whole space, the behavior of the solutions is quite different in the parameter ranges $m_{c}<m<1$ and $0<m<m_{c}$, the critical exponent being defined as

$$
m_{c}:=\frac{d-2}{d}
$$

Note that $m_{c}>0$ only if $d \geq 3$, so that the lower range does not exist for $d=1,2$. For $m>m_{c}$ the mass $\int_{\mathbb{R}^{d}} u(y, t) \mathrm{d} y$ is preserved in time if the initial datum $u_{0}$ is integrable in $\mathbb{R}^{d}$. Besides, non-negative solutions are positive and smooth for all $x \in \mathbb{R}^{d}$ and $t>0$. On the contrary, solutions may extinguish in finite time in the lower range $m<m_{c}$, for instance when the initial data is in $\mathrm{L}^{p_{*}}\left(\mathbb{R}^{d}\right)$ with $p_{*}=d(1-m) / 2$ : then there exists a time $T>0$ such that

$$
\lim _{\tau \nearrow T} u(\tau, y)=0 \text {. }
$$

Many computations are however similar in both ranges, from an algebraic point of view. We refer to the monograph [47] for a detailed discussion of the existence theory and references to the subject. The extension to exponents $m \leq 0$ is also treated, and it is natural but it will not be the focus of this paper.

In the last two decades, special attention has been given to the study of large time asymptotics of these equations, starting with the pioneering work of A. Friedman and S. Kamin [28] and completed in [45], when $m$ is in the range $\left(m_{c}, \infty\right)$. In those studies the class of non-negative,

Date: April 14, 2007.

Key words and phrases. Fast diffusion equation; self-similar solutions; asymptotic behavior; free energy methods; Hardy-Poincaré inequalities - AMS classification (2000): 35B40; 35K55; $39 \mathrm{~B} 62$. 
finite mass solutions are considered. Asymptotic stabilization towards self-similar asymptotic solutions known as Barenblatt solutions is shown. For $m_{c}<m<1$, such solutions take the form:

$$
U_{D, T}(\tau, y):=\frac{1}{R(\tau)^{d}}\left(D+\frac{1-m}{2 m}\left|\frac{y}{R(\tau)}\right|^{2}\right)^{-\frac{1}{1-m}}
$$

with $R(\tau):=\left[d\left(m-m_{c}\right)(\tau+T)\right]^{\frac{1}{d\left(m-m_{c}\right)}}$. Here $D, T \geq 0$ are free parameters. While the second parameter means a time displacement and does not play much role in the asymptotic behavior, the first does and can be computed from the mass of the solution. The value $m_{c}$ is the critical exponent below which the Barenblatt solutions cease to exist in this standard form.

Here, we are mainly interested in addressing the question of the asymptotic behavior of (1.1) when $0<m<m_{c}$. We consider a wide class of solutions which vanish in finite time $T$ and describe their behavior as $\tau$ goes to $T$. We point out that our methods allow to treat simultaneously the ranges $0<m<m_{c}$ and $m_{c} \leq m<1$, in which one is interested in the behavior of the solutions as $\tau$ goes to infinity. For this purpose, we extend the Barenblatt solutions to the range $0<m<m_{c}$ with the same expression (1.2), but a different form for $R$, that is

$$
R(\tau):=\left[d\left(m_{c}-m\right)(T-\tau)\right]^{-\frac{1}{d\left(m_{c}-m\right)}} .
$$

The parameter $T$ now denotes the extinction time. Following [47], we shall call such solutions the pseudo-Barenblatt solutions. Notice that Barenblatt and pseudo-Barenblatt solutions $U_{D, T}$, with $D, T>0$, are such that $U_{D, T}^{p}$ is integrable if and only if $p>p_{*}$ ( $p_{*}$ is defined above, and $p_{*}>1$ means $m<m_{c}$ ). Consistently with the above choices, for $m=m_{c}$, one has to choose $R(\tau):=e^{\tau+T}$ with free parameter $T$, see [47], in order to obtain pseudo-Barenblatt solutions; then, $p_{*}=1$.

The family of Barenblatt (respectively pseudo-Barenblatt) solutions represents the asymptotic patterns to which many other solutions converge for large times if $m>m_{c}$ (respectively as $t$ goes to $T$ if $0<m<m_{c}$ ). We are interested in the class of solutions for which such a convergence takes place and in the rates of convergence. Both questions strongly depend on $m$. Let us emphasize for instance that the Barenblatt solution $U_{D, T}$ is integrable in $y$ for $m>m_{c}$, while the pseudo-Barenblatt solution corresponding to $m \leq m_{c}$ is not integrable. Since much is known in the case $m>m_{c}$, see for instance $[16,25]$ and $[10,11,13,14,15,27,36,45]$ for more complete results, the main novelty of our paper is concerned with the lower range $m \leq m_{c}$, which has several interesting new features. For instance, in the analysis in high space dimensions, that is $d>4$, another critical exponent appears,

$$
m_{*}:=\frac{d-4}{d-2}<m_{c} .
$$

A key property of $m_{*}$ is that the difference of two pseudo-Barenblatt solutions is integrable for $m \in\left(m_{*}, m_{c}\right)$, while it is not integrable for $m \in\left(0, m_{*}\right]$.

The convergence towards Barenblatt and pseudo-Barenblatt solutions is subtle since the solutions converge to zero everywhere. To capture the asymptotic profiles, it is therefore convenient to rescale the solutions and replace the study of intermediate asymptotics by the study of the convergence to stationary solutions in rescaled variables,

$$
t:=\log \left(\frac{R(\tau)}{R(0)}\right) \quad \text { and } \quad x:=\frac{y}{R(\tau)},
$$

with $R$ as above. In these new variables, if $u$ is a solution to (1.1), the function

$$
v(t, x):=R(\tau)^{d} u(\tau, y)
$$


solves a nonlinear Fokker-Planck type equation,

$$
\begin{cases}\partial_{t} v(t, x)=\Delta v^{m}(t, x)+\nabla \cdot(x v(t, x)) & (t, x) \in(0,+\infty) \times \mathbb{R}^{d}, \\ v(0, x)=v_{0}(x) & x \in \mathbb{R}^{d} .\end{cases}
$$

The initial data for (1.1) and for the rescaled equation (1.4) are related by

$$
u_{0}(y)=R(0)^{-d} v_{0}(y / R(0)),
$$

where $R(0)=\left[d\left|m-m_{c}\right| T\right]^{\frac{1}{d\left(m-m_{c}\right)}}$ only depends on $T$. In this formulation, the Barenblatt and pseudo-Barenblatt solutions are transformed into stationary solutions given by

$$
V_{D}(x):=\left(D+\frac{1-m}{2 m}|x|^{2}\right)^{-\frac{1}{1-m}}
$$

where $0<m<1$ and $D>0$ is a free parameter. With a straightforward abuse of language, we say that $V_{D}$ is a Barenblatt profile, including the case $m \leq m_{c}$. The value $D=0$ can also be admitted as a limit case, but the corresponding solution is singular at $x=0$. See [47] for more details. The parameter $T$ has disappeared from the new problem, but it enters in the change of variables. Note that in all cases, $t$ runs from 0 to infinity in these rescaled variables.

Assumptions and main results. We can write the assumptions on the initial conditions in terms of either $u_{0}$ or $v_{0}$. We assume that

(H1) $u_{0}$ is a non-negative function in $\mathrm{L}_{\text {loc }}^{1}\left(\mathbb{R}^{d}\right)$ and that there exist positive constants $T$ and $D_{0}>D_{1}$ such that

$$
U_{D_{0}, T}(0, y) \leq u_{0}(y) \leq U_{D_{1}, T}(0, y) \quad \forall y \in \mathbb{R}^{d} .
$$

(H2) There exist $D_{*} \in\left[D_{1}, D_{0}\right]$ and $f \in \mathrm{L}^{1}\left(\mathbb{R}^{d}\right)$ such that

$$
u_{0}(y)=U_{D_{*}, T}(0, y)+f(y) \quad \forall y \in \mathbb{R}^{d} .
$$

Note that by the Comparison Principle, see Lemma 2.2 below, in the case $m<m_{c}$, (H1) implies that the extinction occurs at time $T$. When $m>m_{*}$, (H2) follows from (H1) since the difference of two Barenblatt solutions is always integrable. For $m \leq m_{*}$, (H2) is an additional restriction. We shall assume throughout this paper that $d \geq 3$ and observe that (H2) has to be taken into account only if $m_{*}>0$, that is, $d \geq 5$.

In terms of $v_{0}$, with $f$ replaced by $R(0)^{-d} f(y / R(0))$, conditions (H1) and (H2) can be rewritten as follows. To avoid more notations, we keep using $f$ in (H2') although it is not the same function as in (H2).

$\left(\mathrm{H} 1^{\prime}\right) v_{0}$ is a non-negative function in $\mathrm{L}_{\text {loc }}^{1}\left(\mathbb{R}^{d}\right)$ and there exist positive constants $D_{0}>D_{1}$ such that

$$
V_{D_{0}}(x) \leq v_{0}(x) \leq V_{D_{1}}(x) \quad \forall x \in \mathbb{R}^{d} .
$$

(H2') There exist $D_{*} \in\left[D_{1}, D_{0}\right]$ and $f \in \mathrm{L}^{1}\left(\mathbb{R}^{d}\right)$ such that

$$
v_{0}(x)=V_{D_{*}}(x)+f(x) \quad \forall x \in \mathbb{R}^{d} .
$$

If $m \in\left(m_{*}, 1\right)$, the map $D \mapsto \int_{\mathbb{R}^{d}}\left(v_{0}-V_{D}\right) \mathrm{d} x$ is continuous, monotone increasing. Hence we can also define a unique $D_{*} \in\left[D_{1}, D_{0}\right]$ such that

$$
\int_{\mathbb{R}^{d}}\left(v_{0}-V_{D_{*}}\right) \mathrm{d} x=0 .
$$

Before stating any result, one more exponent is needed. We define $p(m)$ as the infimum of all positive real numbers $p$ for which two Barenblatt profiles $V_{D_{1}}$ and $V_{D_{2}}$ are such that $\left|V_{D_{1}}-V_{D_{2}}\right|$ belongs to $\mathrm{L}^{p}\left(\mathbb{R}^{d}\right)$ :

$$
p(m):=\frac{d(1-m)}{2(2-m)}
$$


We see that $p(m)>1$ if $m \in\left(0, m_{*}\right), p\left(m_{*}\right)=1$, and $p(m)<1$ if $m>m_{*}$.

We can now state the convergence of $v(t)$ towards a unique Barenblatt profile. For simplicity, we will write $v(t)$ instead of $x \mapsto v(t, x)$ whenever we want to emphasize the dependence in $t$.

Theorem 1.1 (Convergence to the asymptotic profile). Let $d \geq 3, m \in(0,1)$. Consider the solution $v$ of (1.4) with initial data satisfying (H1')-(H2').

(i) For any $m>m_{*}$, there exists a unique $D_{*} \in\left[D_{1}, D_{0}\right]$ such that $\int_{\mathbb{R}^{d}}\left(v(t)-V_{D_{*}}\right) \mathrm{d} x=0$ for any $t>0$. Moreover, for any $p \in(p(m), \infty], \lim _{t \rightarrow \infty} \int_{\mathbb{R}^{d}}\left|v(t)-V_{D_{*}}\right|^{p} \mathrm{~d} x=0$.

(ii) For $m \leq m_{*}, v(t)-V_{D_{*}}$ is integrable, $\int_{\mathbb{R}^{d}}\left(v(t)-V_{D_{*}}\right) \mathrm{d} x=\int_{\mathbb{R}^{d}} f \mathrm{~d} x$ and $v(t)$ converges to $V_{D_{*}}$ in $\mathrm{L}^{p}\left(\mathbb{R}^{d}\right)$ as $t \rightarrow \infty$, for any $p \in(1, \infty]$.

(iii) (Convergence in Relative Error) For any $p \in(d / 2, \infty]$,

$$
\lim _{t \rightarrow \infty}\left\|v(t) / V_{D_{*}}-1\right\|_{p}=0 \text {. }
$$

In case $m>m_{*}$, the value of $D_{*}$ can be computed at $t=0$ as a consequence of the mass balance law $\int_{\mathbb{R}^{d}}\left(v_{0}-V_{D_{*}}\right) \mathrm{d} x=0$, and then the conservation result holds for all $t>0$, see Proposition 2.3 below. On the other hand, in the case $m \leq m_{*}$ the mass balance does not make sense, but $D_{*}$ is determined by Assumption (H2). In this case, the presence of a perturbation $f$ of $V_{D_{*}}$ with nonzero mass, does not affect the asymptotic behavior of the solution at first order.

In a recent paper [22], P. Daskalopoulos and N. Sesum prove some of the results of Theorem 1.1 under similar hypotheses (see [22, Theorem 1.4]). Actually they only prove the $\mathrm{L}^{\infty}$ convergence in case (ii) and the $\mathrm{L}^{1} \cap \mathrm{L}^{\infty}$ convergence in case (i). Our proof was obtained independently and announced in [7]. It is based on entropy estimates and paves the way to the sharper results on convergence with rates, which are the main purpose of the present paper. Assertion (iii) says that the convergence of (i)-(ii) can be improved into a convergence in relative error, in the sense of [45]. Such a strong convergence may look surprising at first sight, but it is a consequence of Assumption (H1'): the tails of $v_{0}$ and $V_{D_{*}}$ have the same behavior as $|x| \rightarrow \infty$.

We can now state our main asymptotic result, on rates of convergence. To state this second result, we need yet another exponent,

$$
q_{*}:=\frac{2 d(1-m)}{2(2-m)+d(1-m)}
$$

and note that $q_{*}>1$ if $m<m_{*}, q_{*}=1$ if $m=m_{*}$, and $q_{*}<1$ if $m>m_{*}$. For any $q>q_{*}$, the function $V_{D_{*}}$ is in $\mathrm{L}^{(2-m) q /(2-q)}\left(\mathbb{R}^{d}\right)$, which allows us to use convenient Hölder interpolation inequalities. We define the $C^{j}$ semi-norm by

$$
\|f\|_{C^{j}\left(\mathbb{R}^{d}\right)}:=\max _{|\eta|=j} \sup _{x \in \mathbb{R}^{d}}\left|\partial^{\eta} f(x)\right|,
$$

where the standard multi-index notation is used: $|\eta|=\eta_{1}+\ldots+\eta_{d}$ is the length of the multiindex $\eta=\left(\eta_{1}, \ldots, \eta_{d}\right) \in \mathbb{Z}^{d}$. The last ingredient is a Hardy-Poincaré constant, which is defined as follows. For any $m \in\left(0, m_{*}\right) \cup\left(m_{*}, 1\right)$, let

$$
\lambda_{m, d}:=m \inf _{h} \frac{\int_{\mathbb{R}^{d}}|\nabla h|^{2} V_{D_{*}} \mathrm{~d} x}{\int_{\mathbb{R}^{d}}|h-\bar{h}|^{2} V_{D_{*}}^{2-m} \mathrm{~d} x},
$$

where the infimum is taken over the set of smooth functions $h$ such that $\operatorname{supp}(h) \subset \mathbb{R}^{d} \backslash\{0\}$ and $\bar{h}=0$ if $m<m_{*}$, while for $m>m_{*}, \bar{h}:=\int_{\mathbb{R}^{d}} h V_{D_{*}}^{2-m} \mathrm{~d} x / \int_{\mathbb{R}^{d}} V_{D_{*}}^{2-m} \mathrm{~d} x$, cf. Theorem A.1 in the Appendix for more details. We shall prove that $\lambda_{m, d}$ is positive and independent of $D_{*}$. In the next result, the time decay rate is formulated in terms of the spectral gap $\lambda_{m, d}$. Analyzing the relationship between the optimal constant $\mathcal{C}_{m, d}=m / \lambda_{m, d}$ in the corresponding functional inequality and the asymptotic rates of the fast diffusion equation is the leitmotiv of this paper. The case $m=m_{*}$ has to be excluded for reasons which are deeply related to Hardy's inequality, see $[7]$. 
Theorem 1.2 (Convergence with rate). Under the assumptions of Theorem 1.1, with $\lambda_{m, d}$ given by (1.6), if $m \neq m_{*}$, there exists $t_{0} \geq 0$ such that the following properties hold:

(i) For any $q \in\left(q_{*}, \infty\right]$, there exists a positive constant $C_{q}$ such that

$$
\left\|v(t)-V_{D_{*}}\right\|_{q} \leq C_{q} \mathrm{e}^{-\lambda_{m, d} t} \quad \forall t \geq t_{0} .
$$

(ii) For any $\vartheta \in[0,(2-m) /(1-m))$, there exists a positive constant $K_{\vartheta}$ such that

$$
\left\||x|^{\vartheta}\left(v(t)-V_{D_{*}}\right)\right\|_{2} \leq K_{\vartheta} \mathrm{e}^{-\lambda_{m, d} t} \quad \forall t \geq t_{0} .
$$

(iii) For any $j \in \mathbb{N}$, there exists a positive constant $H_{j}$ such that

$$
\left\|v(t)-V_{D_{*}}\right\|_{C^{j}\left(\mathbb{R}^{d}\right)} \leq H_{j} \mathrm{e}^{-\frac{2 \lambda_{m, d}}{d+2(j+1)} t} \quad \forall t \geq t_{0} .
$$

The constants $C_{q}, K_{\vartheta}$ and $H_{j}$ depend on $t_{0}, m, d, v_{0}, D_{0}, D_{1}$, and $q, \vartheta$ and $j ; t_{0}$ also depends on $D_{0}$ and $D_{1}$. It is remarkable that the decay rate of the nonlinear problem is given exactly by $\lambda_{m, d}$ (see Section 6.3). Using (1.3), the results of Theorem 1.2 for the solution $v(t)$ of (1.4) can be translated into results for the solution $u(\tau)$ of (1.1) as follows.

Corollary 1.3 (Intermediate asymptotics). Let $d \geq 3, m \in(0,1), m \neq m_{*}$. Consider a solution $u$ of (1.1) with initial data satisfying (H1)-(H2). For $\tau$ large enough, for any $q \in\left(q_{*}, \infty\right]$, there exists a positive constant $C$ such that

$$
\left\|u(\tau)-U_{D_{*}}(\tau)\right\|_{q} \leq C R(\tau)^{-\alpha},
$$

where $\alpha=\lambda_{m, d}+d(q-1) / q$ with $\lambda_{m, d}$ given by (1.6), and large means $T-\tau>0$, small, if $m<m_{c}$, and $\tau \rightarrow \infty$ if $m \geq m_{c}$.

We also obtain a convergence result in relative error. For any $p \in(d / 2, \infty]$, define

$$
\lambda(p):=\frac{(2 p-d)(1-m)}{p(d+2)(2-m)} \lambda_{m, d} .
$$

Theorem 1.4 (Exponential Decay of Relative Error). Under the assumptions of Theorem 1.2, if $m \neq m_{*}$, for any $p \in(d / 2, \infty]$, there exists a positive constant $\mathcal{C}$ and $\lambda \in(0, \lambda(p))$ such that

$$
\left\|v(t) / V_{D_{*}}-1\right\|_{p} \leq \mathcal{C} \mathrm{e}^{-\lambda t} \quad \forall t \geq 0 .
$$

Let us list a few observations on the above results.

(a) In the range $m_{c}<m<1$, convergence with rates has been obtained under various assumptions, cf. $[25,13,15]$ (optimal rates) for $m \in\left[m_{1}, 1\right), m_{1}=(d-1) / d$, and $[14,16]$ for $m \in\left(m_{c}, m_{1}\right)$, which are weaker than the ones of Theorem 1.2. See [27] for the detailed analysis of the spectrum of the linearized operator in the range $m>m_{c}$. A stronger convergence has also been proved in the sense of relative error under very mild assumptions, cf. [45].

(b) In the range $m_{c}<m<1$, Assumption (H1) is less restrictive than one could think. By the global Harnack principle of [11], see Theorem 2.5 below, any solution with non-negative initial data $u_{0} \in \mathrm{L}_{\text {loc }}^{1}\left(\mathbb{R}^{d}\right)$ that decays at infinity like $u_{0}(y)=O\left(|y|^{2 /(1-m)}\right)$, is indeed trapped for all $t>0$ between two Barenblatt solutions if $m_{c}<m<1$. The restrictions on the class of initial data are therefore not so essential as far as the asymptotic behavior is concerned, and can therefore be relaxed.

(c) In the range $0<m \leq m_{c}$, the pseudo-Barenblatt solutions are not integrable. For $m<m_{c}$ many solutions vanish in finite time and have various asymptotic behaviors depending on the initial data. Solutions with bounded and integrable initial data are described by self-similar solutions with so-called anomalous exponents, see [34, 41] and [47, Chapter 7]. Even for solutions with initial data not so far from a pseudo-Barenblatt solution, the asymptotic behavior may significantly differ from the behavior of a pseudo-Barenblatt solution: in [22, Theorem 1.4] a 
solution of (1.1) is found, which is such that $\lim _{|x| \rightarrow \infty} u_{0}(x) / U_{D, T}(0, x)=1$ and which, after rescaling, does not converge to $V_{D}$ as $t \rightarrow \infty$, that is for $\tau \rightarrow T$. Assumption (H1)-(H2) are therefore more restrictive than for $m>m_{c}$.

(d) Proofs are constructive and the values of the various constants are explicit although not so easy to write. The interested reader will be able to recover their expressions by carefully reading the proofs, where all details are given. See Appendix A for more details on the constant $\lambda_{m, d}$ that controls the rate of convergence. The rate given by this exponent is sharp in the linear case, and a deeper analysis should prove that it is sharp also in the nonlinear case. Obtaining the optimal value of $\lambda_{m, d}$ is still an open question in the range $m \in\left(m_{*}, m_{c}\right]$. Our method gives convergence with rates even in the limit case $m=m_{c}$, which is new.

Further comments. After stating our main results, let us come back a little bit on the motivations of this paper, on the main tools and the originality of our results with respect to the existing literature.

During the last few years, asymptotic rates of convergence for the solutions of nonlinear diffusion equations have attracted lots of attention, usually in connection with time-dependent scalings and entropy methods. This has been first done in the range of exponents corresponding to the porous medium equation, with $1<m<2$, and in the range where standard Gagliardo-Nirenberg inequalities apply, $m_{1} \leq m<1$, see $[25,13,15]$. The class of non-negative, finite mass solutions has to be narrowed to the smaller set of functions with finite free energy, or to be precise, with finite entropy and finite potential energy. In the rescaled variables, asymptotic stabilization to the Barenblatt profiles holds at an exponential rate, while in the original time variable $\tau$, the convergence of the difference with the Barenblatt solutions holds at a power-law rate, which is shown to be optimal.

The next question was to understand what happens for $m<m_{1}$. After the linearized analysis of [14], the proof of convergence with rates was done in [16] in the range $m_{c}<m<m_{1}$ for which global existence of finite mass solutions still holds. The basin of attraction is narrowed to the class of solutions with finite relative entropy with respect to some Barenblatt solution.

A dramatic change occurs for $m<m_{c}$, since a large class of solutions vanish in finite time. As a consequence, mass is not conserved, and a key estimate for higher values of $m$ is lost. It is however natural to investigate the basin of attraction of the pseudo-Barenblatt solutions for $m \leq m_{c}$ using relative entropy techniques and to study the convergence rates. This can be done in a weighted space using functional inequalities, which can still be related to some spectral properties of a differential operator obtained by an appropriate linearization.

The generalized entropy functional, or free energy functional, is defined as

$$
\mathcal{E}[v]:=\int_{\mathbb{R}^{d}}\left[\varphi(v)+\frac{1}{2}|x|^{2} v\right] \mathrm{d} x \quad \text { where } \quad \varphi(v):=\frac{v^{m}}{m-1} .
$$

It is then observed that the free energy of the Barenblatt profiles, cf. [15, 25], becomes infinite if $m \leq m_{0}$, where $m_{0}:=d /(d+2) \in\left(m_{c}, m_{1}\right)$. In order to avoid this difficulty, it is convenient to work with the relative entropy of $v$ with respect to $V_{D}$ defined as follows:

$$
\mathcal{E}\left[v \mid V_{D}\right]:=\int_{\mathbb{R}^{d}}\left[\varphi(v)-\varphi\left(V_{D}\right)-\varphi^{\prime}\left(V_{D}\right)\left(v-V_{D}\right)\right] \mathrm{d} x .
$$

The relative entropy is the key tool of our analysis. It is such that $\mathcal{E}\left[v \mid V_{D}\right]:=\mathcal{E}[v]-\mathcal{E}\left[V_{D}\right]$ if $m \in\left(m_{0}, 1\right)$ and $\int_{\mathbb{R}^{d}} v \mathrm{~d} x=\int_{\mathbb{R}^{d}} V_{D} \mathrm{~d} x$, that is for $D=D_{*}$, where $D_{*}$ is as in Theorem 1.1. The functional $\mathcal{E}\left[v \mid V_{D_{*}}\right]$ can also be defined for $m \leq m_{0}$. By homogeneity of $\varphi$, we can indeed rewrite it as

$$
\mathcal{E}\left[v \mid V_{D_{*}}\right]:=\int_{\mathbb{R}^{d}}\left[\varphi(w)-\varphi(1)-\varphi^{\prime}(1)(w-1)\right] V_{D_{*}}^{m} \mathrm{~d} x \quad \text { with } \quad w=\frac{v}{V_{D_{*}}} .
$$


This makes clear why it is well defined at least for $w$ close enough to 1 as $|x| \rightarrow \infty$. The functional $v \mapsto \mathcal{E}\left[v \mid V_{D_{*}}\right]$ is convex and achieves its minimum, 0 , for $v=V_{D_{*}}$. If $v$ is a solution of (1.4), the entropy production term takes the form

$$
-\frac{d}{d t} \mathcal{E}\left[v(t) \mid V_{D_{*}}\right]=\mathcal{I}\left[v(t) \mid V_{D_{*}}\right],
$$

where the functional

$$
v \mapsto \mathcal{I}\left[v \mid V_{D}\right]:=\int_{\mathbb{R}^{d}} v\left|\nabla \varphi^{\prime}(v)-\nabla \varphi^{\prime}\left(V_{D}\right)\right|^{2} \mathrm{~d} x
$$

will be called the relative Fisher information. See Proposition 2.6 for more details. For any $m \in\left[m_{1}, 1\right), \mathcal{E}\left[v \mid V_{D_{*}}\right] \leq \frac{1}{2} \mathcal{I}\left[v \mid V_{D_{*}}\right]$ holds for any smooth function $v$ and the inequality is nothing else than the optimal Gagliardo-Nirenberg inequality, for which equality is achieved precisely by the Barenblatt profiles, see [25]. In such a case,

$$
\mathcal{E}\left[v(t) \mid V_{D_{*}}\right] \leq \mathcal{E}\left[v_{0} \mid V_{D_{*}}\right] e^{-2 t} \quad \forall t \geq 0 .
$$

The limit case $m=m_{1}$ corresponds to the critical Sobolev inequality whose optimal form was established by T. Aubin and G. Talenti in [1,44], while in the limit $m \rightarrow 1$ one recovers Gross' logarithmic Sobolev inequality, see [29, 25]. For $m \in\left[m_{1}, 1\right)$, F. Otto in [40] noticed that (1.4) can be interpreted as the gradient flow of the free energy with respect to the Wasserstein distance. The exponent $m=m_{1}$ is the limit case for which the displacement convexity property holds true.

Pushing the method to the case $0<m<m_{1}$ requires the use of the relative entropy in place of the free energy. The method applies only to a class of initial data which have a finite relative entropy with respect to some Barenblatt profile $V_{D_{*}}$ and satisfy convenient bounds. Mass can be finite in the case $m \in\left(m_{c}, m_{1}\right)$, which was the framework of some earlier studies, see $[14,16]$, or infinite if $m \in\left(0, m_{c}\right)$. Two Barenblatt profiles $V_{D_{0}}$ and $V_{D_{1}}$ have finite relative entropy, i.e. $\mathcal{E}\left[V_{D_{1}} \mid V_{D_{0}}\right]<\infty$ if and only if either $d \leq 4$, or $d \geq 5$ and $m>m_{*}, m_{*}=(d-4) /(d-2)$. Hence, for $d \geq 5, m=m_{*}$ is a threshold not only for defining the relative mass of two pseudo-Barenblatt solutions, but also for defining their relative entropies or for the integrability of $V_{D_{*}}^{2-m}$. Note that $m_{*}<m_{c}$ for all $d \geq 5$. The proof of Theorem 1.2 amounts to prove that the relative entropy $\mathcal{E}\left[v \mid V_{D_{*}}\right]$ decays in time and converges to 0 at an exponential rate when $t \rightarrow \infty$. For $m>\min \left\{0, m_{*}\right\}, \mathcal{E}\left[v \mid V_{D_{*}}\right]$ is well defined under condition (H1'). For $m<m_{*}$, an additional restriction is required, which is precisely the purpose of $\left(\mathrm{H} 2{ }^{\prime}\right)$.

Our approach of course covers the case $m \geq m_{c}$ and we recover some of the results found in $[14,16]$. Some of our results can also be extended to the range $m<0$, but additional technical complications arise, which are still to be studied. In this paper, we leave apart several interesting questions, like the precise study of the case of $m=m_{*}$ or the equation $u_{t}=\Delta \log u$ in dimension $d \geq 2$, see e.g. [20, 21, 42, 48], which is the natural limiting equation to study in the limit $m \rightarrow 0$. Also see $[31,32,33]$ for results which seem closely related to ours, and [26] in the case $m=(d-2) /(d+2)$. In particular we do not use the Bakry-Emery method introduced in $[2]$, on which the results of $[15,13,36,14,16]$ are based. We prove a conservation of relative mass, which allows us to remove the limitation $m>m_{c}$. Neither mass transportation techniques nor Wasserstein distance are needed, although the approach of Section A.3 is not unrelated, see $[8,3,4,38]$.

The paper is organized as follows. In Section 2, we extend the property of mass conservation, which holds only for $m>m_{c}$, to a property of conservation of relative mass, see Proposition 2.3. This selects a unique Barenblatt profile, which governs the asymptotic behavior. We also establish regularity properties of the solutions. From there on we work with the quotient of the solution by the Barenblatt profile. In Section 3, we prove Theorem 1.1 and establish several properties which are used in the sequel. Sections 4 and 5 are respectively devoted to introducting a suitable linearization and to the derivation of entropy - entropy production estimates in the nonlinear 
case, from the corresponding spectral gap estimates for the linearized problem. The proof of Theorem 1.2 is given in Section 6.

Appendix A is devoted to the proofs of spectral gap estimates, that is, of weighted PoincaréHardy inequalities, which have already been studied in [7], and in [14, 27] in the special case $m>m_{c}$. We consider the family of weights of the form $V_{D}$ or $V_{D}^{2-m}, D>0$, that are obtained from the linearization of the relative entropy. In the limit $D \rightarrow 0$, they yield the case corresponding to the weighted $\mathrm{L}^{2}$ norm of the Caffarelli-Kohn-Nirenberg inequalities, $c f .[12,17]$.

A final section, Appendix B, explains how to extend the results of this paper to the fast diffusion with exponents $m \leq 0$. Note that the equation needs to be properly modified. The conclusion is that the results still hold and the proofs need only minor modifications that are indicated.

\section{Basic Estimates}

We establish in this section the main properties of the solutions that will be used in the sequel.

\section{1. $\mathrm{L}^{1}$-contraction and Maximum Principle.}

Lemma 2.1 ( $\mathrm{L}^{1}$-contraction). For any two non-negative solutions $u_{1}$ and $u_{2}$ of (1.1) defined on a time interval $[0, T)$, with initial data in $\mathrm{L}_{\text {loc }}^{1}\left(\mathbb{R}^{d}\right)$, and any two times $t_{1}$ and $t_{2}$ such that $0 \leq t_{1} \leq t_{2}<T$, we have

$$
\int_{\mathbb{R}^{d}}\left|u_{1}\left(t_{2}\right)-u_{2}\left(t_{2}\right)\right| \mathrm{d} x \leq \int_{\mathbb{R}^{d}}\left|u_{1}\left(t_{1}\right)-u_{2}\left(t_{1}\right)\right| \mathrm{d} x .
$$

The above result is well-known to be true for solutions with $\mathrm{L}^{1}$ data, $c f$. [46, Proposition 9.1], even in the stronger form

$$
\int_{\mathbb{R}^{d}}\left[u_{1}\left(t_{2}\right)-u_{2}\left(t_{2}\right)\right]_{+} \mathrm{d} x \leq \int_{\mathbb{R}^{d}}\left[u_{1}\left(t_{1}\right)-u_{2}\left(t_{1}\right)\right]_{+} \mathrm{d} x,
$$

where $[u]_{+}$denotes the positive part of $u$. The result for data in $\mathrm{L}_{\text {loc }}^{1}\left(\mathbb{R}^{d}\right)$ is obtained by approximation, using the uniqueness of solutions to the Cauchy Problem, which has been established in [30]. Note that when the right-hand side is infinite the result applies but there is nothing to prove. As a consequence, we also obtain the following.

Lemma 2.2 (Comparison Principle). For any two non-negative solutions $u_{1}$ and $u_{2}$ of (1.1) on $[0, T), T>0$, with initial data satisfying $u_{01} \leq u_{02}$ a.e, $u_{02} \in \mathrm{L}_{\mathrm{loc}}^{1}\left(\mathbb{R}^{d}\right)$, then we have $u_{1}(t) \leq u_{2}(t)$ for almost every $t \in[0, T)$.

We will see below that under Assumption (H1)-(H2) the solutions are smooth functions, hence the comparison in the previous result holds everywhere in $[0, T) \times \mathbb{R}^{d}$.

2.2. Conservation of relative mass. Mass conservation is used in the range $m>m_{c}$ to determine the parameter $D$ which characterizes the Barenblatt profile $V_{D}$. In the range $m \leq m_{c}$, we can still prove that $\int_{\mathbb{R}^{d}}\left(v(t)-V_{D}\right) \mathrm{d} x$ is conserved for any $t>0$, even if $V_{D} \notin \mathrm{L}^{1}\left(\mathbb{R}^{d}\right)$.

Proposition 2.3. Let $m \in(0,1)$. Consider a solution $u$ of (1.1) with initial data $u_{0}$ satisfying (H1)-(H2). If for some $D>0, \int_{\mathbb{R}^{d}}\left(u_{0}-U_{D, T}(0, \cdot)\right) \mathrm{d} x$ is finite, then

$$
\int_{\mathbb{R}^{d}}\left[u(\tau, x)-U_{D, T}(\tau, x)\right] \mathrm{d} x=\int_{\mathbb{R}^{d}}\left[u_{0}(x)-U_{D, T}(0, x)\right] \mathrm{d} x \quad \forall \tau \in(0, T) .
$$

Proof. In the range $m>m_{c}, u_{0}$ and $U_{D, T}(0, \cdot)$ are integrable and mass conservation of the solutions of (1.1) is well-known. 
Assume next that $m<m_{c}$ and let $\chi$ be a $C^{2}$ function on $\mathbb{R}^{+}$such that $\chi \equiv 1$ on $[0,1], \chi \equiv 0$ on $[2, \infty)$, and $0 \leq \chi \leq 1$ on $[1,2]$. For any $\lambda>0$, take $\phi_{\lambda}(x):=\chi(|x| / \lambda)$ as a test function and denote by $B_{\lambda}$ the ball $B(0, \lambda)$. Then,

$$
\begin{aligned}
\left|\frac{\mathrm{d}}{\mathrm{d} \tau} \int_{\mathbb{R}^{d}}\left[u(\tau)-U_{D, T}(\tau)\right] \phi_{\lambda} \mathrm{d} y\right|= & \left|\int_{\mathbb{R}^{d}}\left[u^{m}(\tau)-U_{D, T}^{m}(\tau)\right] \Delta \phi_{\lambda} \mathrm{d} y\right| \\
= & \left|\int_{B_{2 \lambda} \backslash B_{\lambda}}\left[u^{m}(\tau)-U_{D, T}^{m}(\tau)\right] \Delta \phi_{\lambda} \mathrm{d} y\right| \\
& \leq C \int_{B_{2 \lambda} \backslash B_{\lambda}}\left|u(\tau)-U_{D, T}(\tau)\right| U_{D_{0}, T}^{m-1}(\tau)\left|\Delta \phi_{\lambda}\right| \mathrm{d} y
\end{aligned}
$$

where $C$ is a numerical constant depending on $D, D_{0}, D_{1}$. As $\lambda \rightarrow \infty$, we observe that in $B_{2 \lambda} \backslash B_{\lambda}, U_{D_{0}, T}^{m-1}$ and $\left|\Delta \phi_{\lambda}\right|$ behave like $\lambda^{2}$ and $\lambda^{-2}$, so that $U_{D_{0}, T}^{m-1}(\tau) \Delta \phi$ is bounded uniformly with respect to $\lambda$. The right hand side is therefore bounded by $\int_{B_{2 \lambda} \backslash B_{\lambda}}\left|u(\tau)-U_{D, T}(\tau)\right| \mathrm{d} y$. For any $\tau_{1}, \tau_{2} \in[0, T)$, we write

$$
\left|\int_{\mathbb{R}^{d}}\left[u\left(\tau_{2}\right)-U_{D, T}\left(\tau_{2}\right)\right] \phi_{\lambda} \mathrm{d} y-\int_{\mathbb{R}^{d}}\left[u\left(\tau_{1}\right)-U_{D, T}\left(\tau_{1}\right)\right] \phi_{\lambda} \mathrm{d} y\right| \leq \int_{\tau_{1}}^{\tau_{2}} \int_{B_{2 \lambda} \backslash B_{\lambda}}\left|u-U_{D, T}\right| \mathrm{d} y \mathrm{~d} \tau .
$$

By the $\mathrm{L}^{1}$-contraction principle, see Lemma 2.1, we also know that $\left|u(\tau, y)-U_{D, T}(\tau, y)\right|$ is integrable in $y$, uniformly for all $\tau>0$. The integrability condition implies that the right-hand side goes to zero in the limit $\lambda \rightarrow+\infty$.

The case $m=m_{c}$ is similar, except that there is no extinction time.

In the rescaled variables given by (1.3), relative mass is also conserved. Consider a solution $v$ of (1.4) with initial data $v_{0}$ satisfying (H1')-(H2'). If for some $D>0, \int_{\mathbb{R}^{d}}\left(v_{0}(x)-V_{D}(t, x)\right) \mathrm{d} x$ is finite, then

$$
\int_{\mathbb{R}^{d}}\left[v(t, x)-V_{D}(t, x)\right] \mathrm{d} x=\int_{\mathbb{R}^{d}}\left[v_{0}(x)-V_{D}(t, x)\right] \mathrm{d} x \quad \forall t>0 .
$$

Whenever $m \leq m_{c}$, we recall that pseudo-Barenblatt solutions have infinite mass, that is $\int_{\mathbb{R}^{d}} V_{D_{*}} \mathrm{~d} y=\infty$, but we observe that the difference of two pseudo-Barenblatt solutions is integrable if $m>m_{*}$. In such a case, the parameter $D \in\left[D_{1}, D_{0}\right]$ in Proposition 2.3 can be arbitrary. In the proof, we can moreover estimate $\int_{B_{2 \lambda} \backslash B_{\lambda}}\left|u-U_{D, T}\right| \mathrm{d} y$ by

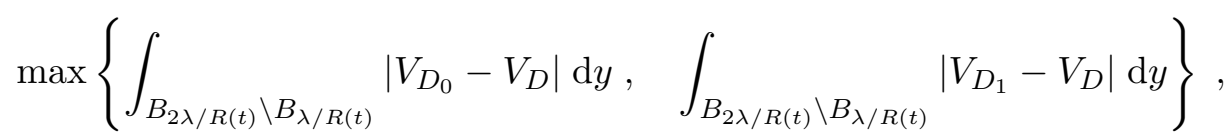

which converges to 0 as $\lambda \rightarrow \infty$. As already quoted in the introduction, the map $D \mapsto \int_{\mathbb{R}^{d}}\left(v_{0}-\right.$ $\left.V_{D}\right) \mathrm{d} x$ is continuous, monotone increasing, and we can define a unique $D_{*} \in\left[D_{1}, D_{0}\right]$ such that $\int_{\mathbb{R}^{d}}\left(v_{0}-V_{D_{*}}\right) \mathrm{d} x=0$. Under the assumptions of Proposition 2.3,

$$
\int_{\mathbb{R}^{d}}\left[v(t, x)-V_{D_{*}}(x)\right] \mathrm{d} x=0 \quad \forall t>0 .
$$

This fact is used in the statement and proof of Theorem 1.1 for $m>m_{*}$. On the contrary, if $m \in\left(0, m_{*}\right]$, integrals are infinite unless $D=D_{*}$ in Proposition 2.3 , and then, with the notations of Assumption (H2'),

$$
\int_{\mathbb{R}^{d}}\left[v(t, x)-V_{D_{*}}(x)\right] \mathrm{d} x=\int_{\mathbb{R}^{d}} f \mathrm{~d} x \quad \forall t>0 .
$$

In both cases, that is for any $m \in(0,1)$, we shall summarize the fact that $\frac{d}{d t} \int_{\mathbb{R}^{d}}\left[v-V_{D_{*}}\right] \mathrm{d} x=0$ by saying that the relative mass is conserved. 
2.3. Passing to the quotient. Consider a solution $v$ of $(1.4)$. As in $[45,11,10]$, define

$$
w(t, x):=\frac{v(t, x)}{V_{D_{*}}(x)} \quad \forall(t, x) \in(0, \infty) \times \mathbb{R}^{d} .
$$

Next, we rewrite Problem (1.4) in terms of $w$ :

$$
\begin{cases}w_{t}=\frac{1}{V_{D_{*}}} \nabla \cdot\left[w V_{D_{*}} \nabla\left(\frac{m}{m-1}\left(w^{m-1}-1\right) V_{D_{*}}^{m-1}\right)\right] & \text { in }(0,+\infty) \times \mathbb{R}^{d}, \\ w(0, \cdot)=w_{0}:=\frac{v_{0}}{V_{D_{*}}} & \text { in } \mathbb{R}^{d} .\end{cases}
$$

Define

$$
W_{0}:=\inf _{x \in \mathbb{R}^{d}} \frac{V_{D_{0}}}{V_{D_{*}}} \leq \sup _{x \in \mathbb{R}^{d}} \frac{V_{D_{1}}}{V_{D_{*}}}:=W_{1} .
$$

A straightforward calculation gives

$$
W_{0}=\left(\frac{D_{*}}{D_{0}}\right)^{\frac{1}{1-m}}<1<\left(\frac{D_{*}}{D_{1}}\right)^{\frac{1}{1-m}}:=W_{1} .
$$

In terms of $w_{0}$, assumptions (H1') and (H2') can be rewritten as follows:

(H1") $w_{0}$ is a non-negative function in $\mathrm{L}_{\text {loc }}^{1}\left(\mathbb{R}^{d}\right)$ and there exist positive constants $D_{0}>D_{1}$ such that

$$
0<W_{0} \leq \frac{V_{D_{0}}(x)}{V_{D_{*}}(x)} \leq w(x) \leq \frac{V_{D_{1}}(x)}{V_{D_{*}}(x)} \leq W_{1}<+\infty \quad \forall x \in \mathbb{R}^{d} .
$$

(H2") There exists $f \in \mathrm{L}^{1}\left(\mathbb{R}^{d}\right)$ such that

$$
w(x)=1+\frac{f(x)}{V_{D_{*}}(x)} \quad \forall x \in \mathbb{R}^{d} .
$$

As a consequence of the Comparison Principle, see Lemma 2.2, (H1") is satisfied by a solution $w$ of $(2.2)$ if it is satisfied by $w_{0}$.

2.4. Regularity estimates and Harnack principle. We start by briefly recalling some wellknown results for solutions of fast diffusion equations. A basic regularity result is due to DiBenedetto et al., see [18, p. 270], and concerns local space-time Hölder regularity for Problem (1.1), with some Hölder exponent $\alpha \in(0,1)$; it holds for locally bounded initial data, possibly with sign changes. In the present situation of locally bounded and positive initial data, it is known that the solutions are $C^{\infty}$ as long as they do not vanish identically because we avoid any degeneracy or singularity and the standard parabolic theory applies. In the sequel we are in particular interested in some sort of uniform $C^{1}$ regularity under the assumption (H1"). We find that it is preferable to work with the function $w$ introduced in (2.1) since it is uniformly bounded from above, and from below away from zero.-

Theorem 2.4 (Uniform $C^{k}$ regularity). Let $m \in(0,1)$ and $w \in \mathrm{L}_{\mathrm{loc}}^{\infty}\left((0, T) \times \mathbb{R}^{d}\right)$ be a solution of (2.2). Then for any $k \in \mathbb{N}$, for any $t_{0} \in(0, T)$,

$$
\sup _{t \geq t_{0}}\|w(t)\|_{C^{k}\left(\mathbb{R}^{d}\right)}<+\infty
$$

Proof. Take $t \geq t_{0}>0$. For a given $\lambda>0$, the equation for $w$ is uniformly parabolic in $B_{\lambda}$, so we conclude that the regularity estimates hold on $B_{\lambda / 2}$ for any $t \geq t_{0}$. Let $v$ be the solution of (1.4) corresponding to $w$. In order to cover the large values of $|x|$, we consider the scaling

$$
v_{\lambda}(x, t)=\lambda^{2 /(1-m)} v(\lambda x, t)
$$


with $\lambda \rightarrow \infty$. Then $v_{\lambda}$ is again a solution of (1.4), but the region $\Omega_{\lambda}=\left\{x \in \mathbb{R}^{d}: \lambda \leq|x| \leq 2 \lambda\right\}$ gets mapped into the region $\Omega_{1}=\left\{x \in \mathbb{R}^{d}: 1 \leq|x| \leq 2\right\}$, for all $\lambda$. Note also that this scaling transforms the Barenblatt profiles according to

$$
\left(V_{D}\right)_{\lambda}=V_{D / \lambda^{2}}
$$

so that, on $\Omega_{1},\left(V_{D}\right)_{\lambda}$ is uniformly bounded from above and from below in $\Omega_{1}$ as $\lambda \rightarrow \infty$, and converges to $V_{0}$.

Next, we pass to the functions $w_{\lambda}(x, t)=v_{\lambda}(x, t) /\left(V_{D_{*}}\right)_{\lambda}(x)$ and observe that in $\Omega_{1},\left(w_{\lambda}\right)_{\lambda \geq 1}$ is uniformly bounded from above, and from below away from zero. Since $w_{\lambda}$ satisfies (2.2) with $V_{D_{*}}$ replaced by $\left(V_{D_{*}}\right)_{\lambda}$, we conclude as in part (i) that

$$
\left\|w_{\lambda}(t)\right\|_{C^{k}\left(\Omega_{1}\right)} \leq C_{k}
$$

uniformly in $t \geq t_{0}$ and $\lambda \geq 1$. Undoing the scaling, we find a constant $C$ independent of $\lambda$ such that, for any $t \geq t_{0}$,

$$
\frac{|\nabla w(t, \lambda x)|}{w(t, \lambda x)} \leq \frac{C}{\lambda}
$$

We conclude that the result holds for $k=1$. The same argument applies for $k>1$.

In the range $m \in\left(m_{c}, 1\right)$, the regularization effects of the fast diffusion equation allow to get rid of assumption (H1). See [11] for details expressions of the constants entering the statement.

Theorem 2.5 (Global Harnack principle, [11, Theorem 1.2]). Let $u_{0}$ be a non-negative function in $\mathrm{L}^{1}\left(\mathbb{R}^{d}\right)$ solution of (1.1). Assume that for some $R>0, \sup _{|y|>R} u_{0}(y)|y|^{2 /(1-m)}$ is finite. Then, for any $\varepsilon>0$, there exist positive constants $T_{0}, T_{1}, D_{0}$ and $D_{1}$, such that

$$
U_{D_{0}, T_{0}}(\tau, y) \leq u(\tau, y) \leq U_{D_{1}, T_{1}}(\tau, y) \quad \forall(\tau, y) \in(\varepsilon, \infty) \times \mathbb{R}^{d}
$$

2.5. Relative entropy. In terms of $w$, we define the relative entropy

$$
\mathcal{F}[w]:=\frac{1}{1-m} \int_{\mathbb{R}^{d}}\left[(w-1)-\frac{1}{m}\left(w^{m}-1\right)\right] V_{D_{*}}^{m} \mathrm{~d} x
$$

and the relative Fisher information

$$
\mathcal{J}[w]:=\frac{m}{(m-1)^{2}} \int_{\mathbb{R}^{d}}\left|\nabla\left[\left(w^{m-1}-1\right) V_{D_{*}}^{m-1}\right]\right|^{2} w V_{D_{*}} \mathrm{~d} x .
$$

These definitions are consistent with the ones given in the introduction, in the sense that, for $w=v / V_{D_{*}}$,

$$
\mathcal{F}[w]=\frac{1}{m} \mathcal{E}\left[v \mid V_{D}\right] \quad \text { and } \quad \mathcal{J}[w]=\frac{1}{m} \mathcal{I}\left[v \mid V_{D}\right] .
$$

The $1 / m$ factor simplifies the expressions of the linearized relative entropy and Fisher information, as we shall see below. It has no impact on the rates. Consistently with the passage to the quotient, the relative entropy and the relative Fisher information are related as follows.

Proposition 2.6. Under Assumptions (H1")-(H2"), if $w$ is a solution of (2.2), then

$$
\frac{d}{d t} \mathcal{F}[w(t)]=-\mathcal{J}[w(t)]
$$

Proof. As in Section 2.2, consider a test function $\phi_{\lambda}(x):=\chi(|x| / \lambda)$ where $\chi$ is a smooth function on $\mathbb{R}^{+}$such that $\chi \equiv 1$ on $[0,1], \chi \equiv 0$ on $[2, \infty)$, and $0 \leq \chi \leq 1$ on $[1,2]$. Then, using (2.2), the 
equality $\left(w^{m-1}-1\right) V_{D_{*}}^{m-1}=v^{m-1}-V_{D_{*}}^{m-1}$ and integration by parts we obtain

$$
\begin{aligned}
\frac{\mathrm{d}}{\mathrm{d} t} & \frac{1}{1-m} \int_{\mathbb{R}^{d}}\left[(w-1)-\frac{1}{m}\left(w^{m}-1\right)\right] \phi_{\lambda} V_{D_{*}}^{m} \mathrm{~d} x \\
& =-\frac{m}{(m-1)^{2}} \int_{\mathbb{R}^{d}} \nabla\left[\left(w^{m-1}-1\right) V_{D_{*}}^{m-1}\right] \cdot \nabla\left[\left(w^{m-1}-1\right) V_{D_{*}}^{m-1} \phi_{\lambda}\right] w V_{D_{*}} \mathrm{~d} x \\
& =-\frac{m}{(m-1)^{2}} \int_{\mathbb{R}^{d}} \nabla\left(v^{m-1}-V_{D_{*}}^{m-1}\right) \cdot\left[\phi_{\lambda} \nabla\left(v^{m-1}-V_{D_{*}}^{m-1}\right)+\nabla \phi_{\lambda}\left(v^{m-1}-V_{D_{*}}^{m-1}\right)\right] v \mathrm{~d} x \\
& =-\frac{m}{(m-1)^{2}} \int_{\mathbb{R}^{d}}\left|\nabla\left(v^{m-1}-V_{D_{*}}^{m-1}\right)\right|^{2} \phi_{\lambda} v \mathrm{~d} x+\mathcal{R}(\lambda),
\end{aligned}
$$

where the last integral can be computed as

$$
\begin{aligned}
\mathcal{R}(\lambda):= & -\frac{m}{2(m-1)^{2}} \int_{\mathbb{R}^{d}} \nabla\left[\left(v^{m-1}-V_{D_{*}}^{m-1}\right)^{2}\right] v \nabla \phi_{\lambda} \mathrm{d} x \\
& =\frac{m}{2(m-1)^{2}} \int_{\mathbb{R}^{d}}\left|v^{m-1}-V_{D_{*}}^{m-1}\right|^{2}\left(\nabla v \cdot \nabla \phi_{\lambda}+v \cdot \Delta \phi_{\lambda}\right) \mathrm{d} x .
\end{aligned}
$$

In the region $\Omega_{\lambda}=\left\{x \in \mathbb{R}^{d}: \lambda \leq|x| \leq 2 \lambda\right\}, \lambda>0$, we get

$$
\begin{aligned}
|\mathcal{R}(\lambda)| & \leq k_{1} \int_{\Omega_{\lambda}}\left|v-V_{D_{*}}\right|^{2} V_{D_{0}}^{2(m-2)}\left(|\nabla v|\left|\nabla \phi_{\lambda}\right|+v\left|\Delta \phi_{\lambda}\right|\right) \mathrm{d} x \\
& \leq k_{1} \sup _{\Omega_{\lambda}}\left[V_{D_{0}}^{2(m-2)}\left|v-V_{D_{*}}\right|\left(|\nabla v|\left|\nabla \phi_{\lambda}\right|+v\left|\Delta \phi_{\lambda}\right|\right)\right] \int_{\Omega_{\lambda}}\left|v-V_{D_{*}}\right| \mathrm{d} x,
\end{aligned}
$$

where the positive constant $k_{1}$ depends on $m, d, D_{0}$ and $D_{1}$.

As in the proof of Theorem 2.4, consider a solution $v$ of (1.4) and define

$$
v_{\lambda}(x, t)=\lambda^{2 /(1-m)} v(\lambda x, t) .
$$

In what follows, $c_{i}$ will denote positive constants which may depend on $m, d, D_{0}, D_{1}$ and on the maximum of $\nabla \phi_{1}$, but not on $\lambda$.

For any $\lambda>0, v_{\lambda}$ is a solution of (1.4) but the region $\Omega_{\lambda}=\left\{x \in \mathbb{R}^{d}: \lambda \leq|x| \leq 2 \lambda\right\}$ gets mapped into the region $\Omega_{1}=\left\{x \in \mathbb{R}^{d}: 1 \leq|x| \leq 2\right\}$. We already know that $\nabla v_{\lambda}$ is uniformly bounded on $\Omega_{1}$, by Theorem 2.4: $\sup _{x \in \Omega_{1}}\left|\nabla v_{\lambda}(t, x)\right| \leq c_{0}$. In terms of $v$, this gives the estimate

$$
\lambda^{\frac{2}{1-m}} \sup _{y \in \Omega_{\lambda}}\left|\nabla_{y} v(t, y)\right|=\lambda^{\frac{2}{1-m}} \sup _{x \in \Omega_{1}}\left|\lambda^{-1} \nabla_{x} v(t, \lambda x)\right|=\lambda^{-1} \sup _{x \in \Omega_{1}}\left|\nabla_{x} v_{\lambda}(t, x)\right| \leq \lambda^{-1} c_{0}
$$

and proves that

$$
\sup _{x \in \Omega_{\lambda}}|\nabla v(t, x)| \leq c_{0} \lambda^{-\frac{2}{1-m}-1} .
$$

By our choice of $\phi_{\lambda}$, we see that

$$
\sup _{\Omega_{\lambda}}\left|\nabla \phi_{\lambda}\right| \leq \frac{c_{1}}{\lambda} \quad \text { and } \quad \sup _{\Omega_{\lambda}}\left|\Delta \phi_{\lambda}\right| \leq \frac{c_{2}}{\lambda^{2}} .
$$

Putting together these two estimates, we get

$$
|\nabla v|\left|\nabla \phi_{\lambda}\right|+v\left|\Delta \phi_{\lambda}\right| \leq c_{3} \lambda^{-\frac{2}{1-m}-2} .
$$

Next, we observe that

$$
-\frac{\partial V_{D}}{\partial D}=\frac{1}{1-m}\left[D+\frac{1-m}{2 m}|x|^{2}\right]^{-\frac{2-m}{1-m}}=\frac{1}{1-m} V_{D}^{2-m} .
$$

Hence, for some constant $c_{4}$ depending on $m, d, D_{0}$ and $D_{1}$,

$$
\left|V_{D_{1}}-V_{D_{0}}\right| \leq c_{4} V_{D_{0}}^{2-m}
$$


and

$$
\sup _{\Omega_{\lambda}}\left[V_{D_{0}}^{2(m-2)}\left|v-V_{D_{*}}\right|\left(|\nabla v|\left|\nabla \phi_{\lambda}\right|+v\left|\Delta \phi_{\lambda}\right|\right)\right] \leq c_{4} \sup _{\Omega_{\lambda}}\left[V_{D_{0}}^{m-2}\left(|\nabla v|\left|\nabla \phi_{\lambda}\right|+v\left|\Delta \phi_{\lambda}\right|\right)\right] .
$$

Taking into account the fact that, for any $\lambda>0, V_{D} \leq c_{5} \lambda^{-2 /(1-m)}$ on $\Omega_{\lambda}$, we obtain

$$
\sup _{\Omega_{\lambda}}\left[V_{D_{0}}^{2(m-2)}\left|v-V_{D_{*}}\right|\left(|\nabla v|\left|\nabla \phi_{\lambda}\right|+v\left|\Delta \phi_{\lambda}\right|\right)\right] \leq c_{6} \lambda^{2 \frac{2-m}{1-m}} \lambda^{-\frac{2}{1-m}-2}=c_{6},
$$

for some positive constant $c_{6}$ which is independent of $\lambda$. By assumptions (H1')-(H2') and the $\mathrm{L}^{1}$-contraction principle, the difference $v-V_{D_{*}}$ is in $\mathrm{L}^{1}$, and so, $\lim _{\lambda \rightarrow \infty} \int_{\Omega_{\lambda}}\left|v-V_{D_{*}}\right| \mathrm{d} x=0$. This proves that $\lim _{\lambda \rightarrow \infty} \mathcal{R}(\lambda)=0$ and we conclude by passing to the limit as $\lambda \rightarrow \infty$.

\section{Convergence Without RAte AND in Relative ERror}

This section is mostly devoted to the proof of Theorem 1.1.

3.1. Relative entropy. Under Assumptions (H1")-(H2"), the relative entropy is well defined.

Lemma 3.1 (An equivalence result). Let $m \in(0,1)$. If $w$ satisfies (H1")-(H2"), then

$$
\frac{1}{2} W_{1}^{m-2} \int_{\mathbb{R}^{d}}|w-1|^{2} V_{D_{*}}^{m} \mathrm{~d} x \leq \mathcal{F}[w] \leq \frac{1}{2} W_{0}^{m-2} \int_{\mathbb{R}^{d}}|w-1|^{2} V_{D_{*}}^{m} \mathrm{~d} x
$$

Proof. For $a>0$, let $\phi_{a}(w):=\frac{1}{1-m}\left[(w-1)-\left(w^{m}-1\right) / m\right]-a(w-1)^{2}$. We compute $\phi_{a}^{\prime}(w)=$ $\frac{1}{1-m}\left[1-w^{m-1}\right]-2 a(w-1)$ and $\phi_{a}^{\prime \prime}(w)=w^{m-2}-2 a$, and note that $\phi_{a}(1)=\phi_{a}^{\prime}(1)=0$. With $a=W_{1}^{m-2} / 2, \phi_{a}^{\prime \prime}$ is positive on $\left(W_{0}, W_{1}\right)$, which proves the lower bound after multiplying by $V_{D}^{m}$ and integrating over $\mathbb{R}^{d}$. With $a=W_{0}^{m-2} / 2, \phi_{a}^{\prime \prime}$ is negative on $\left(W_{0}, W_{1}\right)$ which proves the upper bound.

Lemma 3.2 (Boundedness of the free energy). Let $m \in(0,1)$. If $w_{0}$ satisfies (H1")-(H2"), then the free energy $\mathcal{F}[w(t)]$ is finite for any $t \geq 0$.

Proof. By virtue of Proposition 2.6, we have to prove the result only for $w=w_{0}$. Notice that for any $D_{0}, D_{1}>0$ there exist a positive constant $C$ such that $\left|V_{D_{0}}-V_{D_{1}}\right| \leq C|x|^{-2\left(\frac{2-m}{1-m}\right)}$ as $|x| \rightarrow \infty$. Indeed,

$$
\left|V_{D_{0}}-V_{D_{1}}\right|=\left(\frac{1-m}{2 m}\right)^{-\frac{1}{1-m}} \frac{2 m\left|D_{0}-D_{1}\right|}{(1-m)^{2}}|x|^{-2\left(\frac{2-m}{1-m}\right)}(1+o(1)) \quad \text { as } \quad|x| \rightarrow \infty .
$$

By Lemma 3.1, for some positive constant $c$ depending on $D_{0}$ and $D_{1}$, we have

$$
\frac{2}{W_{0}^{m-2}} \mathcal{F}[w] \leq \int_{\mathbb{R}^{d}}\left|\frac{v}{V_{D_{*}}}-1\right|^{2} V_{D_{*}}^{m} \mathrm{~d} x \leq \int_{\mathbb{R}^{d}}\left|v-V_{D_{*}}\right|^{2} V_{D_{*}}^{m-2} \mathrm{~d} x \leq c \int_{\mathbb{R}^{d}}\left|V_{D_{0}}-V_{D_{1}}\right|^{2} V_{D_{*}}^{m-2} \mathrm{~d} x .
$$

If $m \in\left(m_{*}, 1\right)$, then $\left|V_{D_{0}}-V_{D_{1}}\right|^{2} V_{D_{*}}^{m-2}=O\left(|x|^{-2 \frac{2-m}{1-m}}\right)$ is integrable as $|x| \rightarrow \infty$. Otherwise, if $m \in\left(0, m_{*}\right], \mathcal{F}[w]$ is also integrable as $|x| \rightarrow \infty$ because

$$
\frac{2}{W_{0}^{m-2}} \mathcal{F}[w] \leq \frac{2}{W_{0}^{m-2}} \mathcal{F}\left[w_{0}\right] \leq \int_{\mathbb{R}^{d}}|f|^{2} V_{D_{*}}^{m-2} \mathrm{~d} x \leq \int_{\mathbb{R}^{d}}|f|\left|V_{D_{0}}-V_{D_{1}}\right| V_{D_{*}}^{m-2} \mathrm{~d} x,
$$

$f$ is integrable and $\left|V_{D_{0}}-V_{D_{1}}\right| V_{D_{*}}^{m-2}$ is bounded (we ask the reader to check this fact). 


\subsection{Pointwise convergence in relative error.}

Lemma 3.3. Let $m \in(0,1)$. If $w$ is a solution of (2.2) with initial data $w_{0}$ satisfying (H1")(H2"), then $\lim _{t \rightarrow \infty} w(t, x)=1$ for any $x \in \mathbb{R}^{d}$.

Proof. Let $w_{\tau}(t, x)=w(t+\tau, x)$. By the uniform $C^{k}$ regularity, see Theorem 2.4, the functions $w_{\tau}$ are uniformly $C^{1}$ continuous. Hence, by the Ascoli-Arzelà theorem, there exists a sequence $\tau_{n} \rightarrow \infty$ such that $w_{\tau_{n}}$ converges to a function $w_{\infty}$, locally uniformly in $(t, x)$. We know by the Comparison Principle, see Lemma 2.2, that $w_{\infty}>W_{0}>0$. By interior regularity of the solutions, the derivatives also converge everywhere.

By Lemma $3.2, \mathcal{F}[w]$ is finite. Since

$$
\mathcal{F}\left[w\left(\tau_{n}\right)\right]-\mathcal{F}\left[w\left(\tau_{n}+1\right)\right]=\int_{\tau_{n}}^{\tau_{n}+1} \mathcal{J}[w(s)] \mathrm{d} s=\int_{0}^{1} \mathcal{J}\left[w\left(s+\tau_{n}\right)\right] \mathrm{d} s
$$

as a function of $t, \mathcal{J}\left[w_{\tau_{n}}\right]$ is integrable on $[0,1]$ and converges to zero as $n \rightarrow \infty$,

$$
\lim _{n \rightarrow \infty} \int_{0}^{1} \int_{\mathbb{R}^{d}}\left|\nabla\left[\left(w_{\tau_{n}}^{m-1}(t, x)-1\right) V_{D_{*}}^{m-1}(x)\right]\right|^{2} w_{\tau_{n}}(t, x) V_{D_{*}}(x) \mathrm{d} x \mathrm{~d} t=0 .
$$

By Fatou's lemma, $w_{\infty}=\lim _{n \rightarrow \infty} w_{\tau_{n}}$ satisfies $\nabla\left[\left(w_{\infty}^{m-1}-1\right) V_{D_{*}}^{m-1}\right]=0$ a.e. in $(0,1) \times \mathbb{R}^{d}$. As a consequence of the conservation of relative mass, see Proposition $2.3, w_{\infty}=1$. Thus, we have proved the convergence a.e., and by equi-continuity, the pointwise convergence. Since the limit is unique, the whole family $\left\{w_{\tau}\right\}_{\tau}$ converges everywhere as $\tau \rightarrow \infty$.

\subsection{Proof of Theorem 1.1.}

Proof of Theorem 1.1, (i) and (ii). By Lemma 3.3, $\lim _{t \rightarrow \infty}\left|v(t, x)-V_{D_{*}}(x)\right|=0$ for any $x \in \mathbb{R}^{d}$. Moreover, we observe that

$$
\left|v(t)-V_{D_{*}}\right| \leq \max \left\{\left|V_{D_{0}}-V_{D_{*}}\right|,\left|V_{D_{1}}-V_{D_{*}}\right|\right\}=O\left(|x|^{-2(2-m) /(1-m)}\right)
$$

as $|x| \rightarrow \infty$. By Lebesgue's dominated convergence theorem, $v(t)$ converges to $V_{D_{*}}$ in $\mathrm{L}^{p}\left(\mathbb{R}^{d}\right)$, for any $p \in(p(m), \infty)$, where $p(m):=\frac{d(1-m)}{2(2-m)}$ is the infimum of all positive $p$ such that the difference between two different Barenblatt profiles belongs to $L^{p}\left(\mathbb{R}^{d}\right)$.

The uniform convergence is based on the following interpolation lemma, due to Nirenberg, cf. [39, p. 126]. Let $\lambda, \mu$ and $\nu$ be such that $-\infty<\lambda \leq \mu \leq \nu<\infty$. Then there exists a positive constant $\mathcal{C}_{\lambda, \mu, \nu}$ such that

$$
\|f\|_{1 / \mu}^{\nu-\lambda} \leq \mathcal{C}_{\lambda, \mu, \nu}\|f\|_{1 / \lambda}^{\nu-\mu}\|f\|_{1 / \nu}^{\mu-\lambda} \quad \forall f \in \mathcal{C}\left(\mathbb{R}^{d}\right),
$$

where $\|\cdot\|_{1 / \sigma}$ stands for the following quantities:

(i) If $\sigma>0$, then $\|f\|_{1 / \sigma}=\left(\int_{\mathbb{R}^{d}}|f|^{1 / \sigma} \mathrm{d} x\right)^{\sigma}$.

(ii) If $\sigma<0$, let $k$ be the integer part of $(-\sigma d)$ and $\alpha=|\sigma| d-k$ be the fractional (positive) part of $\sigma$. Using the standard multi-index notation, where $|\eta|=\eta_{1}+\ldots+\eta_{d}$ is the length of the multi-index $\eta=\left(\eta_{1}, \ldots \eta_{d}\right) \in \mathbb{Z}^{d}$, we define

$$
\|f\|_{1 / \sigma}= \begin{cases}\max _{|\eta|=k}\left|\partial^{\eta} f\right|_{\alpha}=\max _{|\eta|=k} \sup _{x, y \in \mathbb{R}^{d}} \frac{\left|\partial^{\eta} f(x)-\partial^{\eta} f(y)\right|}{|x-y|^{\alpha}}=:\|f\|_{C^{\alpha}\left(\mathbb{R}^{d}\right)} & \text { if } \alpha>0, \\ \max _{|\eta|=k} \sup _{z \in \mathbb{R}^{d}}\left|\partial^{\eta} f(z)\right|:=\|f\|_{C^{k}\left(\mathbb{R}^{d}\right)} & \text { if } \alpha=0 .\end{cases}
$$

As a special case, we observe that $\|f\|_{-d / j}=\|f\|_{C^{j}\left(\mathbb{R}^{d}\right)}$.

(iii) By convention, we note $\|f\|_{1 / 0}=\sup _{z \in \mathbb{R}^{d}}|f(z)|=\|f\|_{C^{0}\left(\mathbb{R}^{d}\right)}=\|f\|_{\infty}$. 
Let $j \in \mathbb{N}$ and $\lambda=-(j+1) / d \leq \mu=-j / d \leq \nu=1 / 2$ so that $k=j+1$ and $\alpha=0$. Inequality (3.1) becomes

$$
\|f\|_{C^{j}\left(\mathbb{R}^{d}\right)} \leq \mathcal{C}_{-(j+1) / d,-j / d, 1 / 2}^{\frac{2 d}{d+2(j+1)}}\|f\|_{C^{j+1}\left(\mathbb{R}^{d}\right)}^{\frac{d+2 j}{d+2(j+1)}}\|f\|_{2}^{\frac{2}{d+2(j+1)}}
$$

for any $j \in \mathbb{N}$. By applying this interpolation inequality $f=v(t)-V_{D_{*}}$ with $j=0$, we obtain

$$
\left\|v(t)-V_{D_{*}}\right\|_{\infty} \leq \mathcal{C}_{-1 / d, 0,1 / 2}^{\frac{2 d}{d+2}}\left\|v(t)-V_{D_{*}}\right\|_{C^{1}\left(\mathbb{R}^{d}\right)}^{\frac{d}{d+2}}\left\|v(t)-V_{D_{*}}\right\|_{2}^{\frac{2}{d+2}} .
$$

By Theorem 2.4, the $C^{1}$ norm is uniformly bounded. If $q(m)<2$, that is, if $d \leq 8$, or $d \geq 9$ and $m>(d-8) /(d-4)$, we already know that $\lim _{t \rightarrow \infty}\left\|v(t)-V_{D_{*}}\right\|_{2}=0$. Otherwise, we can interpolate $\left\|v(t)-V_{D_{*}}\right\|_{2}$ between $\left\|v(t)-V_{D_{*}}\right\|_{1} \leq\left\|v_{0}-V_{D_{*}}\right\|_{1}$ (see Lemma 2.1) and $\left\|v(t)-V_{D_{*}}\right\|_{q}$ for some $q>q(m)$. This proves that $\lim _{t \rightarrow \infty}\left\|v(t)-V_{D_{*}}\right\|_{\infty}=0$.

Proof of Theorem 1.1, (iii).

Corollary 3.4 (Uniform convergence of the relative error). Let $m \in(0,1)$. If $w$ is a solution of (2.2) with initial data $w_{0}$ satisfying (H1")-(H2"), then

$$
\lim _{t \rightarrow \infty}\|w(t)-1\|_{\infty}=0 .
$$

Proof. Because of the convergence of $v(t)$ to $V_{D_{*}}$ in $\mathrm{L}^{\infty}\left(\mathbb{R}^{d}\right)$, we know that $w(t)$ converges uniformly to 1 on any compact set of $\mathbb{R}^{d}$. By Assumption (H1'), $v(t)$ is sandwiched between two Barenblatt profiles that have the same asymptotic behavior when $|x|$ is large. In terms of $w$, this means that $|w(t, x)-1|$ is small for $|x|$ large, uniformly in $t$. Global uniform convergence follows.

The fact that $w(t)$ converges uniformly to 1 as $t \rightarrow \infty$ allows us to improve the lower and upper bounds $W_{0}$ and $W_{1}$ for the function $w(t)$, at the price of waiting some time. For any $\varepsilon>0$ there exists a time $t_{0}=t_{0}(\varepsilon) \geq 0$ such that

$$
1-\varepsilon \leq w(t, x) \leq 1+\varepsilon \quad \forall(t, x) \in\left(t_{0}, \infty\right) \times \mathbb{R}^{d} .
$$

Corollary 3.5 ( $\mathrm{L}^{p}$ Convergence of the relative error). Let $m \in(0,1)$. If $w$ is a solution of $(2.2)$ with initial data $w_{0}$ satisfying (H1")-(H2"), then $w(t)$ converges to 1 in $\mathrm{L}^{p}\left(\mathbb{R}^{d}\right)$ for any $p \in$ $(d / 2, \infty]$.

Proof. By Assumptions (H1")-(H2"), there exists a positive constant $c_{0}$ such that $w_{0}-1$ is bounded and for $|x|$ large,

$$
\left|w_{0}-1\right|=\left|\frac{v_{0}-V_{D_{*}}}{V_{D_{*}}}\right| \leq \frac{V_{D_{1}}-V_{D_{0}}}{V_{D_{*}}} \leq \frac{c_{0}}{1+|x|^{2}} .
$$

By Lemma 2.2, the same estimate holds for $w(t)$. Hence $w(t)-1 \in \mathrm{L}^{q}$ for any $q>d / 2$. Let $\delta=(p-d / 2) / 2>0$. By Hölder's inequality,

$$
\lim _{t \rightarrow \infty} \int_{\mathbb{R}^{d}}|w(t)-1|^{p} \mathrm{~d} x \leq \lim _{t \rightarrow \infty}\|w(t)-1\|_{\infty}^{\delta} \int_{\mathbb{R}^{d}}\left(\frac{c_{0}}{1+|x|^{2}}\right)^{\delta+d / 2} \mathrm{~d} x=0 .
$$

Theorem 1.1, (iii), results from Corollaries 3.4 and 3.5. 


\subsection{Uniform convergence and $C^{\alpha}$ regularity.}

Lemma 3.6. Let $m \in(0,1)$. Consider a solution $v$ of (1.4) with initial data $v_{0}$ satisfying (H1')$(\mathrm{H} 2)$. There exists $t_{0} \geq 0, \alpha \in(0,1)$ and a positive constant $\mathcal{H}$ such that $h(t):=v(t)-V_{D_{*}}$ is in $C^{\alpha}$ and

$$
\|h(t)\|_{C^{\alpha}\left(\mathbb{R}^{d}\right)} \leq \mathcal{H}\|h(t)\|_{\infty} \quad \forall t \geq t_{0} .
$$

Proof. Since both $v$ and $V_{D_{*}}$ are solutions to equation (1.4), $h$ solves

$$
h_{t}=\nabla \cdot\left[m\left(V_{D_{*}}+h\right)^{m-1} \nabla h+m\left(\left(V_{D_{*}}+h\right)^{m-1}-V_{D_{*}}^{m-1}-V_{D_{*}}^{m-2} h\right) \nabla V_{D_{*}}\right] .
$$

Let $\lambda>0$. By Theorem 1.1, we know that for some $t_{0} \geq 0$, for any $t \geq t_{0},\|h(t)\|_{\infty}$ can be taken uniformly small and $v$ uniformly positive on $B_{2 \lambda}$. We apply the standard quasilinear parabolic theory, see e.g. [35, Theorem 1.1, p. 418], with structure functions $a_{i}(x, t, h, \xi)=A \xi+B h$ and $a=0$, where $A(x, t):=m v^{m-1}, B(x, t):=m\left[\left(\frac{v^{m-1}-V_{D_{*}}^{m-1}}{v-V_{D_{*}}}-V_{D_{*}}^{m-2}\right) \nabla V_{D_{*}}\right]$. Hence there exists a Hölder exponent $\alpha \in(0,1)$ and a constant $\mathcal{H}$ depending on the uniform bounds for the coefficients, and on $\lambda$, such that (3.4) is verified in $B_{\lambda} \times\left(t_{0}+1, \infty\right)$. To extend the estimate uniformly to the whole space, $x \in \mathbb{R}^{d}$, we use the same scaling argument as in the proof of Theorem 2.4. We leave the details to the reader.

\section{Linearization}

In order to better understand the asymptotic behavior of the solutions of (2.2), we linearize the equation around the equilibrium, introducing a convenient weight. Let $g$ be such that

$$
w(t, x)=1+\varepsilon \frac{g(t, x)}{V_{D_{*}}^{m-1}(x)} \quad \forall t>0, \quad \forall x \in \mathbb{R}^{d},
$$

for some $\varepsilon>0$, small. Substituting this expression in Equation (2.2) and letting $\varepsilon \rightarrow 0$, we formally obtain a linear equation for $g$,

$$
g_{t}=A_{m} g \quad \text { where } \quad A_{m} g:=m V_{D_{*}}^{m-2}(x) \nabla \cdot\left[V_{D_{*}} \nabla g\right] .
$$

The linear operator $A_{m}: \mathrm{L}^{2}\left(\mathbb{R}^{d}, V_{D_{*}}^{2-m} \mathrm{~d} x\right) \rightarrow \mathrm{L}^{2}\left(\mathbb{R}^{d}, V_{D_{*}}^{2-m} \mathrm{~d} x\right)$ is the positive self-adjoint operator associated to the closure of the quadratic form defined for $\phi \in C_{c}^{\infty}\left(\mathbb{R}^{d}\right)$ by

$$
\mathrm{I}[\phi]:=m \int_{\mathbb{R}^{d}}|\nabla g|^{2} V_{D_{*}} \mathrm{~d} x
$$

See [23, Theorem 2.6] for more details.

With the same heuristics, we linearize the relative entropy $\mathcal{F}$ and the relative Fisher information $\mathcal{J}$, which provides the functionals $\mathrm{F}$ and $\mathrm{I}$, where $\mathrm{I}$ is given by (4.3) and $\mathrm{F}$ is defined by

$$
\mathrm{F}[g]:=\frac{1}{2} \int_{\mathbb{R}^{d}}|g|^{2} V_{D_{*}}^{2-m} \mathrm{~d} x .
$$

Note that $\mathrm{F}[g]$ is the $\mathrm{L}^{2}\left(\mathbb{R}^{d}, V_{D_{*}}^{2-m} \mathrm{~d} x\right)$-norm up to a factor $1 / 2$. If $g$ is a solution of $(4.2)$, then

$$
\frac{\mathrm{d}}{\mathrm{d} t} \mathrm{~F}[g(t)]=-\mathrm{I}[g(t)] .
$$

Still at a formal level, the conservation of relative mass amounts to require

$$
\int_{\mathbb{R}^{d}}\left(v_{0}-V_{D_{*}}\right) \mathrm{d} x=\int_{\mathbb{R}^{d}}(w-1) V_{D_{*}} \mathrm{~d} x=\varepsilon \int_{\mathbb{R}^{d}} g V_{D_{*}}^{2-m} \mathrm{~d} x
$$


in the limit $\varepsilon \rightarrow 0$. Hence, it makes sense to require that $\int_{\mathbb{R}^{d}} g V_{D_{*}}^{2-m} \mathrm{~d} x=0$ and use the spectral gap estimate, see [7] and Theorem A.1. With $\mathcal{C}_{m, d}=m / \lambda_{m, d}$, we obtain

$$
2 \mathrm{~F}[g] \leq \frac{\mathcal{C}_{m, d}}{m} \mathrm{I}[g]
$$

which gives, for the solution of (4.1), an exponential decay of the relative entropy,

$$
\mathrm{F}[g(t)] \leq \mathrm{e}^{-2 \lambda_{m, d} t} \mathrm{~F}[g(0)] \quad \forall t \geq 0 .
$$

In Sections 5 and 6, we will compare the relative entropy estimates for the solutions of $(2.2)$ with the ones of the linearized problem. This is the main ingredient of the proof of Theorem 1.2.

The connection with the Fokker-Planck equation is easy to understand at the level of the linearized problem. In the limit $m \rightarrow 1$, we observe that

$$
\lim _{m \rightarrow 1^{-}} D_{*}^{1 /(1-m)} V_{D_{*}}=\left(2 \pi D_{*}\right)^{d / 2} \mu \quad \text { with } \quad \mu(x)=\frac{\mathrm{e}^{-\frac{|x|^{2}}{2 D_{*}}}}{\left(2 \pi D_{*}\right)^{d / 2}} .
$$

Equation (4.2) formally converges to the Ornstein-Uhlenbeck equation,

$$
g_{t}=\mu^{-1} \nabla \cdot(\mu \nabla g) \text {. }
$$

The spectral gap inequality (4.6) corresponds in such a limit to the well-known Poincaré inequality with gaussian weight,

$$
\int_{\mathbb{R}^{d}}|\phi|^{2} \mathrm{~d} \mu \leq \int_{\mathbb{R}^{d}}|\nabla \phi|^{2} \mathrm{~d} \mu \quad \forall \phi \in C^{\infty}\left(\mathbb{R}^{d}\right) \text { such that } \int_{\mathbb{R}^{d}} \phi \mathrm{d} \mu=0,
$$

where $\mathrm{d} \mu:=\mu \mathrm{d} x$. Note that in the Gaussian case, a logarithmic Sobolev inequality holds, see [29],

$$
\int_{\mathbb{R}^{d}}|\phi|^{2} \log \left(\frac{|\phi|^{2}}{\int_{\mathbb{R}^{d}}|\phi|^{2} \mathrm{~d} \mu}\right) \mathrm{d} \mu \leq 2 \int_{\mathbb{R}^{d}}|\nabla \phi|^{2} \mathrm{~d} \mu
$$

which is stronger than the Gaussian Poincaré inequality. This is not the case with the measure $V_{D_{*}} \mathrm{~d} x$. Although the spectral gap inequality (4.6) holds true, there is no corresponding logarithmic Sobolev inequality.

\section{More on the Relative Fisher information}

In this section, we relate the relative Fisher and linearized Fisher informations. This and Lemma 3.1 provide us with an estimate of the relative entropy in terms of the relative Fisher information, or entropy - entropy production inequality, for the nonlinear problem.

\subsection{Fisher information and linearized Fisher information.}

Lemma 5.1 (Upper bound on the Fisher information). Let $m \in(0,1)$. There exists two positive constants $\beta_{1}$ and $\beta_{2}$ (depending on $W_{0}, W_{1}$ and $m$ ) such that, for any $w$ satisfying (H1")-(H2"),

$$
\mathrm{I}[g] \leq \beta_{1} \mathcal{J}[w]+\beta_{2} \mathrm{~F}[g] \quad \text { with } \quad g:=(w-1) V_{D_{*}}^{m-1} .
$$

Moreover, if $\eta:=\max \left\{1-W_{0}, W_{1}-1\right\}$, then $\lim _{\eta \rightarrow 0_{+}}\left(\left|1-\beta_{1}\right|+\beta_{2}\right)=0$.

The constant $\beta_{1}$ and $\beta_{2}$ are explicitly given in (5.2) in terms of $m, D_{*}, W_{0}$ and $W_{1}$.

Proof. Define $h_{k}(w):=\left(w^{k-1}-1\right) /(k-1)$. Let $\alpha_{0}:=W_{0}^{2(2-m)}, \alpha_{1}:=W_{1}^{2(2-m)}$. Since $\left|h_{2} / h_{m}\right|$ is non-decreasing,

$$
\alpha_{0} \leq\left|\frac{h_{2}^{\prime}\left(W_{0}\right)}{h_{m}^{\prime}\left(W_{0}\right)}\right|^{2} \leq\left|\frac{h_{2}(w)}{h_{m}(w)}\right|^{2} \leq\left|\frac{h_{2}\left(W_{1}\right)}{h_{m}\left(W_{1}\right)}\right|^{2} \leq\left|\frac{h_{2}^{\prime}\left(W_{1}\right)}{h_{m}^{\prime}\left(W_{1}\right)}\right|^{2}=\alpha_{1} .
$$


Note that $\alpha_{0}=\alpha_{0}\left(W_{0}\right)<1<\alpha_{1}=\alpha_{1}\left(W_{1}\right)$ and both converge to 1 as $W_{0}, W_{1} \rightarrow 1$.

Using the fact that $V_{D_{*}}^{m-1}=D_{*}+\frac{1-m}{2 m}|x|^{2}$ and an integration by part, we get

$$
\begin{aligned}
& \int_{\mathbb{R}^{d}}\left|\nabla\left[h_{k}(w) V_{D_{*}}^{m-1}\right]\right|^{2} V_{D_{*}} \mathrm{~d} x \\
& =\int_{\mathbb{R}^{d}}\left|h_{k}^{\prime}(w)\right|^{2}|\nabla w|^{2} V_{D_{*}}^{2 m-1} \mathrm{~d} x+\frac{1-m}{m^{2}} \int_{\mathbb{R}^{d}}|x|^{2}\left|h_{k}(w)\right|^{2} V_{D_{*}} \mathrm{~d} x-d \frac{1-m}{m} \int_{\mathbb{R}^{d}}\left|h_{k}(w)\right|^{2} V_{D_{*}}^{m} \mathrm{~d} x .
\end{aligned}
$$

Let $g:=(w-1) V_{D_{*}}^{m-1}$. Applied with $k=2$ and $k=m$, the above identity gives

$$
\begin{aligned}
\mathrm{I}[g]=m \int_{\mathbb{R}^{d}} \mid \nabla & {\left.\left[h_{2}(w) V_{D_{*}}^{m-1}\right]\right|^{2} V_{D_{*}} \mathrm{~d} x } \\
\leq m \alpha_{1} & \int_{\mathbb{R}^{d}}\left|h_{m}^{\prime}(w)\right|^{2}|\nabla w|^{2} V_{D_{*}}^{2 m-1} \mathrm{~d} x \\
& \quad+\alpha_{1} \frac{1-m}{m} \int_{\mathbb{R}^{d}}|x|^{2}\left|h_{m}(w)\right|^{2} V_{D_{*}} \mathrm{~d} x-d(1-m) \int_{\mathbb{R}^{d}}\left|h_{2}(w)\right|^{2} V_{D_{*}}^{m} \mathrm{~d} x
\end{aligned}
$$

and

$$
\begin{aligned}
\int_{\mathbb{R}^{d}}\left|h_{m}^{\prime}(w)\right|^{2}|\nabla w|^{2} & V_{D_{*}}^{2 m-1} \mathrm{~d} x \\
=\int_{\mathbb{R}^{d}} \mid \nabla & {\left.\left[h_{m}(w) V_{D_{*}}^{m-1}\right]\right|^{2} V_{D_{*}} \mathrm{~d} x } \\
& \quad-\frac{1-m}{m^{2}} \int_{\mathbb{R}^{d}}|x|^{2}\left|h_{m}(w)\right|^{2} V_{D_{*}} \mathrm{~d} x+d \frac{1-m}{m} \int_{\mathbb{R}^{d}}\left|h_{m}(w)\right|^{2} V_{D_{*}}^{m} \mathrm{~d} x .
\end{aligned}
$$

Collecting these estimates, we obtain

$$
\mathrm{I}[g] \leq m \alpha_{1} \int_{\mathbb{R}^{d}}\left|\nabla\left[h_{m}(w) V_{D_{*}}^{m-1}\right]\right|^{2} V_{D_{*}} \mathrm{~d} x+d(1-m) \int_{\mathbb{R}^{d}}\left(\alpha_{1}\left|h_{m}(w)\right|^{2}-\left|h_{2}(w)\right|^{2}\right) V_{D_{*}}^{m} \mathrm{~d} x .
$$

Note that

$$
m \int_{\mathbb{R}^{d}}\left|\nabla\left[h_{m}(w) V_{D_{*}}^{m-1}\right]\right|^{2} V_{D_{*}} \mathrm{~d} x \leq W_{0}^{-1} \mathcal{J}[w] .
$$

Using $\mathrm{F}[g]=\frac{1}{2} \int_{\mathbb{R}^{d}}|g|^{2} V_{D_{*}}^{2-m} \mathrm{~d} x=\frac{1}{2} \int_{\mathbb{R}^{d}}\left|h_{2}(w)\right|^{2} V_{D_{*}}^{m} \mathrm{~d} x$ with $g:=(w-1) V_{D_{*}}^{m-1}$, we obtain

$$
\mathbf{I}[g] \leq \beta_{1} \mathcal{J}[w]+\beta_{2} \mathbf{F}[w],
$$

with

$$
\beta_{1}:=\frac{\alpha_{1}}{W_{0}}=\frac{W_{1}^{2(2-m)}}{W_{0}} \quad \text { and } \quad \beta_{2}:=2 d(1-m)\left(\frac{\alpha_{1}}{\alpha_{0}}-1\right) .
$$

Note that $\alpha_{0}=\alpha_{0}\left(W_{0}\right)<1<\alpha_{1}=\alpha_{1}\left(W_{1}\right)$ and both tend to 1 as $W_{0}, W_{1} \rightarrow 1$.

\subsection{Entropy - entropy production inequality.}

Theorem 5.2 (Entropy - entropy production inequality). Let $m \in(0,1), m \neq m_{*}$. For any function $w=w(x)$ satisfying (H1")-(H2"), if $1-W_{0}>0$ and $W_{1}-1>0$ are small enough, then there exists a positive constant $\gamma$ such that

$$
\gamma \mathcal{F}[w] \leq \mathcal{J}[w] .
$$

As we shall see in the proof, the constant $\gamma$ can be estimated as follows:

$$
\gamma \geq 2 \frac{m-\mathcal{C}_{m, d} d(1-m)\left[\left(\frac{W_{1}}{W_{0}}\right)^{2(2-m)}-1\right]}{\mathcal{C}_{m, d} W_{0}^{m-3} W_{1}^{2(2-m)}} .
$$


The condition that $1-W_{0}>0$ and $W_{1}-1>0$ are small enough in the statement of Theorem 5.2 can be replaced by a weaker condition which amounts to ask that the right hand side of the above estimate is positive, that is, with $\lambda_{m, d}=m / \mathcal{C}_{m, d}$,

$$
\frac{W_{1}}{W_{0}}<\left(1+\frac{\lambda_{m, d}}{d(1-m)}\right)^{\frac{1}{2(2-m)}} .
$$

Proof. Let $g:=(w-1) V_{D_{*}}^{m-1}$. By definition of $D_{*}, 0=\int_{\mathbb{R}^{d}}(w-1) V_{D_{*}} \mathrm{~d} x=\int_{\mathbb{R}^{d}} g V_{D_{*}}^{2-m} \mathrm{~d} x$ if $m>m_{*}$. By the spectral gap estimate (4.6) (also see Theorem A.1),

$$
2 \mathrm{~F}[g] \leq \frac{\mathcal{C}_{m, d}}{m} \mathrm{I}[g] .
$$

By Lemma 5.1,

$$
2 \mathrm{~F}[g] \leq \frac{\mathcal{C}_{m, d}}{m} \mathrm{I}[g] \leq \frac{\mathcal{C}_{m, d}}{m}\left(\beta_{1} \mathcal{J}[w]+\beta_{2} \mathrm{~F}[g]\right),
$$

from which we deduce that

$$
\mathrm{F}[g] \leq \frac{\mathcal{C}_{m, d} \beta_{1}}{2 m-\mathcal{C}_{m, d} \beta_{2}} \mathcal{J}[w] .
$$

By Lemma 3.1, the conclusion holds with

$$
\gamma=\frac{2 m-\mathcal{C}_{m, d} \beta_{2}}{W_{0}^{m-2} \mathcal{C}_{m, d} \beta_{1}}
$$

under the condition $2 m-\mathcal{C}_{m, d} \beta_{2}>0$. According to the definition (5.2) of $\beta_{2}$, this amounts to the condition

$$
\mathcal{C}_{m, d} \beta_{2}=2 d(1-m) \mathcal{C}_{m, d}\left(\frac{\alpha_{1}}{\alpha_{0}}-1\right)<2 m
$$

that is $\alpha_{1} / \alpha_{0}$ close enough to 1 , which follows from the requirement that $1-W_{0}>0$ and $W_{1}-1>0$ are small enough.

\section{Convergence with Rates}

\subsection{Exponential decay of the relative entropy.}

Proposition 6.1. Let $m \in(0,1), m \neq m_{*}$. There exists a positive constant $\gamma$ such that, for any solution $w$ of (2.2) with initial data $w_{0}$ satisfying (H1")-(H2"), if $1-W_{0}>0$ and $W_{1}-1>0$ are small enough, then

$$
\mathcal{F}[w(t)] \leq \mathcal{F}\left[w_{0}\right] \mathrm{e}^{-\gamma t} .
$$

The value of $\gamma$ can be estimated from below by (5.3).

Proof. We combine formula $\frac{d}{d t} \mathcal{F}[w(t)]=-\mathcal{J}[w(t)]$ with the estimate $\mathcal{J}[w(t)] \geq \gamma \mathcal{F}[w(t)]$ obtained in Theorem 5.2, and then integrate the resulting differential inequality.

6.2. Moments, $\mathrm{L}^{p}$ and $C^{k}$ estimates. We recall that $q_{*}:=\frac{2 d(1-m)}{d(1-m)+2(2-m)}$ and define

$$
\gamma(q):=\frac{1}{2} \text { if } q \in\left(q_{*}, 2\right], \quad \gamma(q)=\frac{q+d}{q(d+2)} \text { if } q \in(2, \infty), \quad \gamma(\infty):=\frac{1}{d+2} .
$$

The following lemma helps to understand better the consequences of the convergence of the free energy $\mathcal{E}\left[v \mid V_{D_{*}}\right]$, in terms of $\mathrm{L}^{p}$, moment and also $C^{k}$ convergence.

Lemma 6.2. Let $m \in(0,1)$ and consider a function $v$ satisfying (H1')-(H2'). Then 
(i) For any $\vartheta \in\left[0, \frac{2-m}{1-m}\right]$, there exists a positive constant $K_{\vartheta}$ such that

$$
\left\||x|^{\vartheta}\left(v-V_{D_{*}}\right)\right\|_{2} \leq K_{\vartheta}\left(\mathcal{E}\left[v \mid V_{D_{*}}\right]\right)^{1 / 2} .
$$

(ii) For any $q \in\left(q_{*}, 2\right]$, there exists a positive constant $K(q)$ such that

$$
\left\|v-V_{D_{*}}\right\|_{q} \leq K(q)\left(\mathcal{E}\left[v \mid V_{D_{*}}\right]\right)^{\gamma(q)} .
$$

Consider now a solution $v$ of (1.4) such that $v_{0}$ satisfies (H1')-(H2') and fix some $t_{0}>0$. Then

(iii) For any $j \in \mathbb{N}$ and any $t_{0}>0$, there exists a positive constant $H_{j}$ such that

$$
\left\|v(t)-V_{D_{*}}\right\|_{C^{j}\left(\mathbb{R}^{d}\right)} \leq H_{j}\left(\mathcal{E}\left[v(t) \mid V_{D_{*}}\right]\right)^{\frac{1}{d+2(j+1)}} \quad \forall t \geq t_{0} .
$$

(iv) For any $q \in(2, \infty]$, there exists a positive constant $K(q)$ such that

$$
\left\|v(t)-V_{D_{*}}\right\|_{q} \leq K(q)\left(\mathcal{E}\left[v(t) \mid V_{D_{*}}\right]\right)^{\gamma(q)} \quad \forall t \geq t_{0} .
$$

Proof. (i) With $\kappa_{\vartheta}:=2 \sup _{r>0} r^{2 \vartheta}\left(D_{*}+\frac{1-m}{2 m} r^{2}\right)^{-(2-m) /(1-m)}$,

$$
\left\||x|^{\vartheta}\left(v-V_{D_{*}}\right)\right\|_{2}^{2} \leq \frac{1}{2} \kappa_{\vartheta} \int_{\mathbb{R}^{d}}\left|v-V_{D_{*}}\right|^{2} V_{D_{*}}^{m-2} \mathrm{~d} x \leq \kappa_{\vartheta} \mathrm{F}\left[(w-1) V_{D_{*}}^{m-1}\right],
$$

and the right hand side is equivalent to $\mathcal{E}\left[v \mid V_{D_{*}}\right]$ by Lemma 3.1.

(ii) Let $q \in\left(q_{*}, 2\right)$. By Hölder's inequality,

$\int_{\mathbb{R}^{d}}\left|v-V_{D_{*}}\right|^{q} \mathrm{~d} x=\int_{\mathbb{R}^{d}} V_{D_{*}}^{(2-m) q / 2} \cdot\left(\left|v-V_{D_{*}}\right|^{2} V_{D_{*}}^{m-2}\right)^{q / 2} \mathrm{~d} x \leq c(q)\left(\int_{\mathbb{R}^{d}}\left|v-V_{D_{*}}\right|^{2} V_{D_{*}}^{m-2} \mathrm{~d} x\right)^{q / 2}$,

where $c(q)^{2 /(2-q)}:=\int_{\mathbb{R}^{d}} V_{D_{*}}^{(2-m) \frac{q}{2-q}} \mathrm{~d} x$ is finite for any $q>q_{*}$. By Lemma 3.1 , the estimate holds with $K(q):=c(q)^{1 / q}\left(2 W_{1}^{2-m}\right)^{1 / 2}$. In the limit case $q=2$, the same method applies with $c(2)=\left\|V_{D_{*}}^{(2-m)}\right\|_{\infty}=D_{*}^{-(2-m) /(1-m)}$.

(iii) We apply the interpolation inequality (3.2) to $f=v(t)-V_{D_{*}}$ and bound $\left\|v(t)-V_{D_{*}}\right\|_{C^{j}\left(\mathbb{R}^{d}\right)}$ in terms of $\|v(t)\|_{C^{j+1}}$, which is uniformly bounded by Theorem 2.4 and $\left\|v(t)-V_{D_{*}}\right\|_{2}^{2 /(d+2(j+1))}$, for which we apply the result of Part (i) with $\vartheta=0$.

(iv) By Theorem 2.4, $v(t) \in C^{1}\left(\mathbb{R}^{d}\right)$ and $v(t)-V_{D_{*}}$ is bounded in $C^{1}$ uniformly for any $t \geq t_{0}>0$. By (3.3),

$$
\left\|v(t)-V_{D_{*}}\right\|_{\infty} \leq \mathcal{C}_{-1 / d, 0,1 / 2}^{\frac{2 d}{d+2}}\left\|v(t)-V_{D_{*}}\right\|_{C^{1}\left(\mathbb{R}^{d}\right)}^{\frac{d}{d+2}}\left\|v(t)-V_{D_{*}}\right\|_{2}^{\frac{2}{d+2}}
$$

We conclude by using Hölder's inequality, $\left\|v(t)-V_{D_{*}}\right\|_{q} \leq\left\|v(t)-V_{D_{*}}\right\|_{\infty}^{(q-2) / q}\left\|v(t)-V_{D_{*}}\right\|_{2}^{2 / q}$.

\subsection{Improvement of the convergence.}

Theorem 6.3. Let $d \geq 3, m \in(0,1)$ with $m \neq m^{*}$. Consider a solution $w$ of $(2.2)$ with initial data satisfying (H1")-(H2"). There exist a positive constant $\mathcal{K}$ and a time $t_{0} \geq 0$ such that

$$
\mathcal{F}[w(t)] \leq \mathcal{K} \mathrm{e}^{-2 \lambda_{m, d} t} \quad \forall t \geq t_{0} .
$$

Moreover, for any $\lambda \in\left(0, \lambda_{m, d}\right)$, there exist a positive constant $\mathcal{C}_{\infty}$ and a time $t_{0} \geq 0$ such that

$$
\|w(t)-1\|_{\infty} \leq \mathcal{C}_{\infty} \mathrm{e}^{-2 \frac{1-m}{2-m} \frac{\lambda}{d+2} t} \quad \forall t \geq t_{0} .
$$


Here $\lambda_{m, d}=m / \mathcal{C}_{m, d}$ where $\mathcal{C}_{m, d}$ is given in Theorem A.1. Hence the rate of decay obtained by spectral methods for the linearized equation exactly gives the rate of decay for the nonlinear problem, and the price to be paid is only on the constant $\mathcal{K}$. As a subproduct of the proof, for some positive constants $\eta_{0}$ and $\gamma_{\infty}$ which are defined below, we obtain the following estimate

$$
\mathcal{K} \leq \mathcal{F}\left[w\left(t_{0}\right)\right] \mathrm{e}^{\frac{\eta_{0}(2-m)}{\gamma_{\infty}(1-m)}} \mathrm{e}^{2 \lambda_{m, d} t_{0}}
$$

Proof. By Corollary 3.4, for any $\varepsilon>0$, there exists $t_{0}>0$ such that $\tilde{w}(t)=w\left(t+t_{0}\right)$ satisfies Assumption (H2") at $t=0$ with $0<1-W_{0}<\varepsilon$ and $0<W_{1}-1<\varepsilon$. With $\varepsilon$ small enough, $\tilde{w}$ enters in the framework of Proposition 6.1, with $\gamma$ as in Theorem 5.2. From now on, we assume that $t \geq t_{0}$ and simply write $w$ instead of $\tilde{w}$.

On the one hand, by Lemma 6.2 and Proposition 6.1, we have

$$
\left\|v(t)-V_{D_{*}}\right\|_{\infty} \leq \sigma_{0} \mathrm{e}^{-\gamma_{\infty}\left(t-t_{0}\right)} \quad \text { with } \quad \sigma_{0}:=K(\infty) \mathcal{F}\left[w_{0}\right]^{\frac{1}{d+2}} \quad \text { and } \quad \gamma_{\infty}=\frac{\gamma}{d+2},
$$

which, in terms of $w$, gives the estimate

$$
|w(t, x)-1| \leq \sigma_{0} \mathrm{e}^{-\gamma_{\infty}\left(t-t_{0}\right)}\left[D_{*}+\frac{1-m}{2 m}|x|^{2}\right]^{\frac{1}{1-m}} \quad \forall t \geq t_{0}, \quad \forall x \in \mathbb{R}^{d} .
$$

On the other hand, let $h_{\alpha}(s):=(1+s)^{\alpha}, \alpha>1$. For any $s \in\left[0, s_{0}\right]$,

$$
\frac{h_{\alpha}(s)-1}{s} \leq \alpha+\frac{s_{0}}{2} \max _{s \in\left[0, s_{0}\right]} h_{\alpha}^{\prime \prime}(s)= \begin{cases}\alpha+\frac{s_{0}}{2} \alpha(\alpha-1)\left(1+s_{0}\right)^{\alpha-2} & \text { if } \quad \alpha \geq 2, \\ \alpha+\frac{s_{0}}{2} \alpha(\alpha-1) & \text { if } \quad \alpha \leq 2 .\end{cases}
$$

Apply then this inequality with $\alpha=1 /(1-m), s=\left(D_{*}-D_{1}\right) /\left(D_{1}+\frac{1-m}{2 m}|x|^{2}\right) \leq s_{0}=\frac{D_{*}}{D_{1}}-1$ to get the existence of a positive constant $\mathcal{M}_{1}=\mathcal{M}_{1}\left(m, D_{*}, D_{1}\right)$ such that

$$
w(t, x)-1 \leq \frac{V_{D_{1}}}{V_{D_{*}}}-1=\left(1+\frac{D_{*}-D_{1}}{D_{1}+\frac{1-m}{2 m}|x|^{2}}\right)^{\frac{1}{1-m}}-1 \leq \frac{\mathcal{M}_{1}}{D_{1}+\frac{1-m}{2 m}|x|^{2}} \quad \forall x \in \mathbb{R}^{d} .
$$

Similarly, for any $s \in\left[-s_{0}, 0\right]$,

$$
\frac{h_{\alpha}(s)-1}{s} \geq \alpha-\frac{s_{0}}{2} \max _{s \in\left[-s_{0}, 0\right]} h_{\alpha}^{\prime \prime}(s)= \begin{cases}\alpha-\frac{s_{0}}{2} \alpha(\alpha-1) & \text { if } \quad \alpha \geq 2, \\ \alpha-\frac{s_{0}}{2} \alpha(\alpha-1)\left(1-s_{0}\right)^{\alpha-2} & \text { if } \quad \alpha \leq 2,\end{cases}
$$

so that, with $\alpha=1 /(1-m), s=-\left(D_{0}-D_{*}\right) /\left(D_{0}+\frac{1-m}{2 m}|x|^{2}\right) \leq-s_{0}=\frac{D_{*}}{D_{0}}-1$, we get the existence of a positive constant $\mathcal{M}_{0}=\mathcal{M}_{0}\left(m, D_{*}, D_{0}\right)$ such that

$$
w(t, x)-1 \geq \frac{V_{D_{0}}}{V_{D_{*}}}-1=\left(1-\frac{D_{0}-D_{*}}{D_{0}+\frac{1-m}{2 m}|x|^{2}}\right)^{\frac{1}{1-m}}-1 \geq \frac{\mathcal{M}_{0}}{D_{0}+\frac{1-m}{2 m}|x|^{2}} \quad \forall x \in \mathbb{R}^{d} .
$$

Hence, there exists a positive constant $\mathcal{M}$ depending on $\max \left\{\mathcal{M}_{0}, \mathcal{M}_{1}\right\}, D_{0}$ and $D_{1}$, for which we obtain

$$
|w(t, x)-1| \leq \min \left\{\frac{\sigma_{0} \mathrm{e}^{-\gamma_{\infty}\left(t-t_{0}\right)}}{V_{D_{*}}}, \mathcal{M} V_{D_{*}}^{1-m}\right\}=\sigma \mathrm{e}^{-\gamma_{\infty} \frac{1-m}{2-m}\left(t-t_{0}\right)}, \quad \sigma:=\mathcal{M}^{\frac{1}{2-m}} \sigma_{0}^{\frac{1-m}{2-m}} .
$$

As a consequence, for any $t \geq t_{0}$, we have improved bounds on $w$, with $W_{0}$ and $W_{1}$ replaced respectively by $\sigma_{0}(t):=1-\sigma \mathrm{e}^{-\gamma_{\infty} \frac{1-m}{2-m}\left(t-t_{0}\right)}$ and $\sigma_{1}(t):=1+\sigma \mathrm{e}^{-\gamma_{\infty} \frac{1-m}{2-m}\left(t-t_{0}\right)}$. As in Proposition 6.1, according to Theorem 5.2 and Inequality $(5.3), z(t)=\mathcal{F}[w(t)]$ satisfies

$$
\frac{d z}{d t} \leq-\gamma(t) z(t)
$$


with

$$
\gamma(t):=2 \frac{m \sigma_{0}(t)^{2(2-m)}-\mathcal{C}_{m, d} d(1-m)\left[\sigma_{1}(t)^{2(2-m)}-\sigma_{0}(t)^{2(2-m)}\right]}{\mathcal{C}_{m, d} \sigma_{0}(t)^{1-m} \sigma_{1}(t)^{2(2-m)}}=2 \lambda_{m, d}-\eta(t),
$$

$\lambda_{m, d}=m / \mathcal{C}_{m, d}$ and $\eta(t) \leq \eta_{0} \mathrm{e}^{-\gamma_{\infty} \frac{1-m}{2-m}\left(t-t_{0}\right)}$ for some $\eta_{0}>0$. A Gronwall argument then shows that for any $t \geq t_{0}$,

$\log \left(\frac{z(t)}{z\left(t_{0}\right)}\right) \leq-2 \lambda_{m, d}\left(t-t_{0}\right)+\frac{\eta_{0}}{\gamma_{\infty} \frac{1-m}{2-m}}\left[1-\mathrm{e}^{-\gamma_{\infty} \frac{1-m}{2-m}\left(t-t_{0}\right)}\right] \leq-2 \lambda_{m, d}\left(t-t_{0}\right)+\frac{\eta_{0}(2-m)}{\gamma_{\infty}(1-m)}$.

which completes the estimate on $\mathcal{F}[w(t)]$. For $t$ large enough, $\frac{1}{2} \gamma(t) \in\left(\lambda, \lambda_{m, d}\right)$ and the $\mathrm{L}^{\infty}$ estimate follows.

Proof of Theorem 1.4. As in the proof of Corollary 3.5, a Hölder interpolation inequality shows that, for any $\delta>0$,

$$
\int_{\mathbb{R}^{d}}|w(t)-1|^{p} \mathrm{~d} x \leq\|w(t)-1\|_{\infty}^{p-\delta-\frac{d}{2}} \int_{\mathbb{R}^{d}}\left(\frac{c_{0}}{1+|x|^{2}}\right)^{\delta+\frac{d}{2}} \mathrm{~d} x
$$

6.4. Proof of Theorem 1.2. We first apply Theorem 6.3 and Lemma 6.2 , (i), with $\vartheta=0$ to obtain, for some $t_{0} \geq 0$,

$$
\left\|v(t)-V_{D_{*}}\right\|_{2} \leq K_{\vartheta=0}\left(\mathcal{E}\left[v(t) \mid V_{D_{*}}\right]\right)^{\frac{1}{2}} \leq C_{2} \mathrm{e}^{-\lambda_{m, d} t} \quad \forall t \geq t_{0},
$$

for some positive constant $C_{2}$. By the interpolation inequality (3.1) with $\lambda=-\alpha d<0=\mu<$ $1 / 2=\nu, C=\mathcal{C}_{-\alpha d, 0,1 / 2}$, and Lemma 3.6, (3.4), we have

$$
\left\|v(t)-V_{D_{*}}\right\|_{\infty} \leq C\left\|v(t)-V_{D_{*}}\right\|_{C^{\alpha}}^{\theta}\left\|v(t)-V_{D_{*}}\right\|_{2}^{1-\theta} \leq C \mathcal{H}^{\theta}\left\|v(t)-V_{D_{*}}\right\|_{\infty}^{\theta}\left\|v(t)-V_{D_{*}}\right\|_{2}^{1-\theta}
$$

where $\theta=1 /(2+\alpha d)$. This implies

$$
\left\|v(t)-V_{D_{*}}\right\|_{\infty} \leq C^{1 /(1-\theta)} \mathcal{H}^{\theta /(1-\theta)}\left\|v(t)-V_{D_{*}}\right\|_{2} \quad \forall t \geq t_{0} .
$$

From Hölder's inequality, $\left\|v(t)-V_{D_{*}}\right\|_{q} \leq\left\|v(t)-V_{D_{*}}\right\|_{\infty}^{(q-2) / q}\left\|v(t)-V_{D_{*}}\right\|_{2}^{2 / q}, q \in(2, \infty]$, we deduce that $\left\|v(t)-V_{D_{*}}\right\|_{q}$ decays with the same rate as $\left\|v(t)-V_{D_{*}}\right\|_{2}$. If $q \in\left(q_{*}, 2\right)$, we apply Lemma 6.2, (ii), and Theorem 6.3 to prove that for some positive constant $C_{q}$ and for some $t_{0} \geq 0$

$$
\left\|v(t)-V_{D_{*}}\right\|_{q} \leq C_{q} \mathrm{e}^{-\lambda_{m, d} t} \quad \forall t \geq t_{0} .
$$

Similarly, the estimate $\left\|v(t)-V_{D_{*}}\right\|_{C^{j}\left(\mathbb{R}^{d}\right)}$ follows from Lemma 6.2, (iii), and Theorem 6.3. This completes the proof of Theorem 1.2.

\section{Appendix A: Hardy-Poincaré inequalities}

In this appendix, we state and prove a result on inequalities which we have already been partially studied in [7]. Here we give more details and a few improvements. We are especially interested in the explicit values of the constants which enter in the convergence rates of Theorems 1.2 and 1.4. This is why we take weights which are adapted to Equation (1.4) and define the measures

$$
d \mu:=V_{D}^{2-m} \mathrm{~d} x \quad \text { and } \quad d \nu:=V_{D} \mathrm{~d} x,
$$

where $V_{D}(x)=\left(D+\frac{1-m}{2 m}|x|^{2}\right)^{-1 /(1-m)}$. Incidentally we observe that $d \mu=V_{D}^{1-m} d \nu$. To a function $g \in \mathrm{L}^{1}(d \mu)$, we associate its average $\bar{g}=\int_{\mathbb{R}^{d}} g(x) d \mu$. Recall that $m_{*}=(d-4) /(d-2)$. 


\section{A.1. Statement and comments.}

Theorem A.1. Let $d \geq 1$ and $D>0$. If $m \in(0,1)$ and $1 \leq d \leq 4$, or $m \in\left(m_{*}, 1\right)$ and $d \geq 5$, then there exists a positive constant $\mathcal{C}_{m, d}$, which does not depend on $D$, such that

$$
\int_{\mathbb{R}^{d}}|g-\bar{g}|^{2} d \mu \leq \mathcal{C}_{m, d} \int_{\mathbb{R}^{d}}|\nabla g|^{2} d \nu \quad \forall g \in \mathcal{D}\left(\mathbb{R}^{d}\right), \quad \bar{g}=\int_{\mathbb{R}^{d}} g d \mu .
$$

In case $d \geq 5$ and $m \in\left(0, m_{*}\right)$, we have

$$
\int_{\mathbb{R}^{d}} g^{2} d \mu \leq \mathcal{C}_{m, d} \int_{\mathbb{R}^{d}}|\nabla g|^{2} d \nu \quad \forall g \in \mathcal{D}\left(\mathbb{R}^{d}\right)
$$

and $\mathcal{C}_{m, d}=\frac{8 m(1-m)}{\left[(d-2)\left(m-m_{*}\right)\right]^{2}}$ is optimal.

Estimates of the optimal constant $\mathcal{C}_{m, d}$ when $m>m_{*}$ are given below in Proposition A.3. With $v_{m}(x)=\left(1+|x|^{2}\right)^{-1 /(1-m)}$, a simple change of variables shows that $\lambda_{m, d}=m / \mathcal{C}_{m, d}$ is such that

$$
\lambda_{m, d}=m \inf _{h} \frac{\int_{\mathbb{R}^{d}}|\nabla h|^{2} V_{D} \mathrm{~d} x}{\int_{\mathbb{R}^{d}}|h-\bar{h}|^{2} V_{D}^{2-m} \mathrm{~d} x}=\frac{1-m}{2} \inf _{h} \frac{\int_{\mathbb{R}^{d}}|\nabla h|^{2} v_{m} \mathrm{~d} x}{\int_{\mathbb{R}^{d}}|h-\tilde{h}|^{2} v_{m}^{2-m} \mathrm{~d} x},
$$

where the infima are taken over the set of smooth functions $h$ such that

- either $m<m_{*}$ and $\operatorname{supp}(h) \subset \mathbb{R}^{d} \backslash\{0\}$ and $\bar{h}=0, \tilde{h}=0$,

- or $m>m_{*}$,

$$
\bar{h}:=\frac{\int_{\mathbb{R}^{d}} h V_{D}^{2-m} \mathrm{~d} x}{\int_{\mathbb{R}^{d}} V_{D}^{2-m} \mathrm{~d} x} \quad \text { and } \quad \tilde{h}:=\frac{\int_{\mathbb{R}^{d}} h v_{m}^{2-m} \mathrm{~d} x}{\int_{\mathbb{R}^{d}} v_{m}^{2-m} \mathrm{~d} x} .
$$

This already shows that $\lambda_{m, d}$ is independent of $D$.

We observe that as $|x| \rightarrow \infty, d \mu \sim d \nu /|x|^{2}$. Hence, if $m \in\left(0, m_{*}\right)$, Inequality (A.2) is of Hardy type. Otherwise, if $m \in\left(m_{*}, 1\right)$, Inequality (A.1) involves an average and is rather of Poincaré type. In such a case, we shall also say that it is a weighted Poincaré inequality, or that there is a spectral gap, since for the associated operator, the lowest eigenvalue, 0 , is achieved by the constant functions, and the second eigenvalue corresponds to $\lambda_{m, d}=m / \mathcal{C}_{m, d}$ where $\mathcal{C}_{m, d}$ is the best constant in the inequality. See [7] for further considerations on these issues.

We also remark that Theorem A.1 provides an explicit example for which the weighted Poincaré inequality holds, while the corresponding weighted logarithmic Sobolev inequality does not hold, even in dimension $d=1$, as shown by [3, Theorem 3].

The proofs of (A.1) and (A.2) are quite different and for this reason we treat the two cases separately. We start with the proof of (A.2) corresponding to the case $m<m_{*}, d \geq 5$.

A.2. Case $m \in\left(0, m_{*}\right)$. The proof follows the ideas of [7]. We reproduce it here for completeness. We compute

and

$$
\left|\nabla V_{D}(x)\right|^{2}=\frac{|x|^{2}}{m^{2}} V_{D}(x)^{2(2-m)}
$$

$$
-2 m^{2} \frac{\Delta V_{D}(x)}{V_{D}(x)^{3-2 m}}=2 d D m+(d-2)\left(m_{*}-m\right)|x|^{2} .
$$

An integration by parts and the Cauchy-Schwarz inequality show that

$$
\begin{aligned}
\left.\left|\int_{\mathbb{R}^{d}}\right| g\right|^{2} \Delta V_{D} \mathrm{~d} x \mid & \leq 2 \int_{\mathbb{R}^{d}}|g||\nabla g|\left|\nabla V_{D}\right| \mathrm{d} x \\
& \leq 2\left(\int_{\mathbb{R}^{d}}|g|^{2}\left|\Delta V_{D}\right| \mathrm{d} x\right)^{1 / 2}\left(\int_{\mathbb{R}^{d}}|\nabla g|^{2}\left|\nabla V_{D}\right|^{2}\left|\Delta V_{D}\right|^{-1} \mathrm{~d} x\right)^{1 / 2} .
\end{aligned}
$$


As in [24], we remark that $\Delta V_{D}$ has a constant sign and get the estimate

$$
\left.\left.\left|\int_{\mathbb{R}^{d}}\right| g\right|^{2} \Delta V_{D} \mathrm{~d} x\left|=\int_{\mathbb{R}^{d}}\right| g\right|^{2}\left|\Delta V_{D}\right| \mathrm{d} x \leq 4 \int_{\mathbb{R}^{d}}|\nabla g|^{2}\left|\nabla V_{D}\right|^{2}\left|\Delta V_{D}\right|^{-1} \mathrm{~d} x .
$$

Weights can be estimated on both sides of the inequality:

$$
\begin{aligned}
& \frac{\left|\Delta V_{D}\right|}{V_{D}^{2-m}}=\frac{2 d D m+(d-2)\left(m_{*}-m\right)|x|^{2}}{m\left(2 D m+(1-m)|x|^{2}\right)} \geq \frac{(d-2)\left(m_{*}-m\right)}{m(1-m)}, \\
& \frac{\left|\nabla V_{D}\right|^{2}}{\left|\Delta V_{D}\right| V_{D}} \leq \frac{2|x|^{2}}{2 d D m+(d-2)\left(m_{*}-m\right)|x|^{2}} \leq \frac{2}{(d-2)\left(m_{*}-m\right)},
\end{aligned}
$$

which proves (A.2). See [7] for further details.

We now consider the limit $D \rightarrow 0^{+}$. With $\alpha:=1 /(m-1) \in(1-d / 2,-1)$, that is $m \in\left(0, m_{*}\right)$, and

$$
\kappa_{\alpha}:=\frac{8 m(1-m)}{\left[(d-2)\left(m-m_{*}\right)\right]^{2}} \cdot \frac{1-m}{2 m}=\frac{4(1-m)^{2}}{[(d-4)-(d-2) m]^{2}},
$$

Inequality (A.2) takes the form of a weighted Hardy inequality,

$$
\int_{\mathbb{R}^{d}} \frac{|g|^{2}}{|x|^{2}}|x|^{\alpha} \mathrm{d} x \leq \kappa_{\alpha} \int_{\mathbb{R}^{d}}|\nabla g|^{2}|x|^{\alpha} \mathrm{d} x \quad \forall g \in \mathcal{D}\left(\mathbb{R}^{d}\right) .
$$

Such an inequality is easy to establish by the "completing the square method" as follows. Let $\alpha \in \mathbb{R} \backslash\{\alpha\}$ with $\alpha_{*}:=1-d / 2$, and $g \in \mathcal{D}\left(\mathbb{R}^{d}\right)$. Then

$$
\begin{aligned}
0 \leq & \int_{\mathbb{R}^{d}}\left|\nabla g+\lambda \frac{x}{|x|^{2}} g\right|^{2}|x|^{2 \alpha} \mathrm{d} x \\
& =\int_{\mathbb{R}^{d}}|\nabla g|^{2}|x|^{2 \alpha} \mathrm{d} x+\left[\lambda^{2}-\lambda(2 \alpha+d-2)\right] \int_{\mathbb{R}^{d}} \frac{|g|^{2}}{|x|^{2}}|x|^{2 \alpha} \mathrm{d} x .
\end{aligned}
$$

An optimization of the right hand side with respect to $\lambda$ results in choosing $\lambda=(2 \alpha+d-2) / 2$, that is

$$
\frac{1}{\lambda^{2}}=\frac{4}{(d+2 \alpha-2)^{2}}=\frac{4(1-m)^{2}}{[(d-4)-(d-2) m]^{2}}=\kappa_{\alpha}
$$

The weighted Hardy inequality is optimal, with optimal constant $\kappa_{\alpha}$, as follows by considering the test functions $g_{\varepsilon}(x):=\min \left\{\varepsilon^{-\lambda},\left(|x|^{-\lambda}-\varepsilon^{\lambda}\right)_{+}\right\}$and letting $\varepsilon \rightarrow 0$.

A closer inspection of the proof reveals that the constant $\kappa_{\alpha}$ in the weighted Hardy inequality also is optimal when $m>m_{*}$. Consider indeed the test functions $g_{\varepsilon}(x):=|x|^{1-\alpha-d / 2+\varepsilon}$ for $|x|<1$ and $g_{\varepsilon}(x)=(2-|x|)_{+}$for $|x| \geq 1$, and then let $\varepsilon \rightarrow 0$.

Proposition A.2 (Weighted Hardy inequality). With the above notations, for any $\alpha \in \mathbb{R}$, $\alpha \neq \alpha_{*}$,

$$
\int_{\mathbb{R}^{d}} \frac{|g|^{2}}{|x|^{2}}|x|^{2 \alpha} \mathrm{d} x \leq \kappa_{\alpha} \int_{\mathbb{R}^{d}}|\nabla g|^{2}|x|^{2 \alpha} \mathrm{d} x \quad \forall g \in \mathcal{D}\left(\mathbb{R}^{d}\right),
$$

with the additional requirement that $g$ is supported in $\mathbb{R}^{d} \backslash\{0\}$ if $\alpha<\alpha_{*}$, and $\kappa_{\alpha}$ is optimal.

The range $m \in(0,1)$ corresponds to $1 /(m-1)=\alpha \in(-\infty,-1)$, so that $m=m_{*}$ is equivalent to $\alpha=\alpha_{*}$. Notice that the result holds without other restriction than $\alpha \neq \alpha_{*}$, but one has to be careful with integrability condition at $x=0$ if $\alpha<\alpha_{*}$. 
A.3. Case $\max \left\{0, m_{*}\right\}<m<1$. Several partial results are known. In the range $m \in\left(m_{c}, 1\right)$, see [14] for an estimate of $\mathcal{C}_{m, d}$ based on the Bakry-Emery method, [7] for other estimates, and [27] for the exact values of the optimal constant for a corresponding linear problem.

We now prove (A.1) with some explicit estimates of the constant $\mathcal{C}_{m, d}$ in the whole range $\left(\max \left\{0, m_{*}\right\},(d-2) /(d-1)\right) \supset\left(m_{*}, m_{c}\right]$. Because of the change of variables (A.3), our task is now to characterize $\mathcal{C}_{m, d}$ as

$$
\left(\frac{(1-m) \mathcal{C}_{m, d}}{2 m}\right)^{-1}=\inf _{h} \frac{\int_{\mathbb{R}^{d}}|\nabla h|^{2} v_{m} \mathrm{~d} x}{\int_{\mathbb{R}^{d}}|h-\tilde{h}|^{2} v_{m}^{2-m} \mathrm{~d} x} .
$$

On $\mathbb{R}^{+}$, consider the function $\mu(r):=r^{d-1}\left(1+|r|^{2}\right)^{(2-m) /(m-1)}$, and denote its median by $\eta$. Let $\nu(r):=r^{d-1}\left(1+r^{2}\right)^{1 /(m-1)}$ and define for all $\zeta>0$ the quantity

$$
\mathrm{K}(\zeta):=\frac{2 m}{1-m} \max \{\mathrm{A}(\zeta), \mathrm{B}(\zeta)\}
$$

with

$$
\mathrm{A}(\zeta):=\sup _{r<\zeta}\left[\int_{0}^{r} \mu(s) \mathrm{d} s \int_{r}^{\zeta} \frac{\mathrm{d} s}{\nu(s)}\right], \quad \mathrm{B}(\zeta):=\sup _{r>\zeta}\left[\int_{\zeta}^{r} \frac{\mathrm{d} s}{\nu(s)} \int_{r}^{+\infty} \mu(s) \mathrm{d} s\right] .
$$

By convention, we take $\mathrm{K}(0)=\frac{2 m}{1-m} \mathrm{~B}(0)$. The following result is inspired by $[3,8,19,38]$.

Proposition A.3. Let $d \geq 1$. For any $m \neq m_{*}$,

$$
\mathcal{C}_{m, d} \geq \frac{8 m(1-m)}{[d-4-m(d-2)]^{2}} .
$$

If $m \in\left(m_{*}, 1\right)$, then

$$
\mathcal{C}_{m, 1} \leq \mathrm{K}(0) \quad \text { and } \quad \mathcal{C}_{m, d} \leq \max \left\{2 \mathrm{~K}(\eta), \frac{4 m}{(1-m)(d-1)}\right\} \quad \text { if } \quad d \geq 2,
$$

where, for any $m \in\left(m_{*},(d-2) /(d-1)\right)$,

$$
\mathrm{K}(\eta) \leq \frac{m(2-m) 2^{\frac{3-2 m}{1-m}}\left(1+2^{\frac{2-m}{1-m}}\right)}{d[d-4-m(d-2)]^{2}} .
$$

The function $v_{m}^{2-m}$ is integrable for any $m \in\left(m_{*}, 1\right)$, so that $\mathrm{K}(\eta)$ is well defined in this range. The upper bound on $\mathcal{C}_{m, d}$ is equal to its exact value up to a factor which is at least $1 / 4$ (and at most 1). Such an interval is inherent to the method, see [38]. The case $m=m_{c} \leq(d-2) /(d-1)$ is covered, showing in particular that $\mathcal{C}_{m_{c}, d}$ is positive, finite. The bounds diverge as $m \searrow m^{*}$ with same behavior at first order. Our approach can be extended easily to the case $((d-2) /(d-1), 1)$, with slightly different estimates of $\mathrm{K}(\eta)$, but this case is already covered in $[7,14,27]$ by other methods. The restriction $m<(d-2) /(d-1)$ is convenient from a technical point of view, and not essential at all.

Proof. The lower bound on $\mathcal{C}_{m, d}$ is achieved as in Section A.2 by taking the limit $D=0$, thus showing that $\mathcal{C}_{m, d} \geq \frac{1-m}{2 m} \kappa_{1 /(m-1)}$ and using Proposition A.2.

Let us prove the upper bounds. We introduce the standard change of variables from Cartesian to spherical coordinates, i.e. $r=|x|$, and $\vartheta=x /|x|$. In these coordinates, the gradient can be written as $\left(\partial_{r}, \frac{1}{r} \nabla_{\theta}\right)$ where $\partial_{r}=\frac{x}{r} \cdot \nabla$ is the partial derivative with respect to the radial variable $r$ and $\nabla_{\theta}$ is the derivative with respect to the angular variables. We shall denote by $\mathbb{S}^{d-1} \subset \mathbb{R}^{d}$ the unit sphere and parametrize it with the variable $\vartheta$. 
The radial density functions $r \mapsto \mu(r)$ and $r \mapsto \nu(r)$ are such that $v_{m} \mathrm{~d} x=\mu(|x|) \mathrm{d} x$ and $v_{m}^{2-m} \mathrm{~d} x=\nu(|x|) \mathrm{d} x$. We introduce the following normalization constants:

$$
\omega_{d}=\int_{\mathbb{S}^{d-1}} \mathrm{~d} \vartheta=\frac{2 \pi^{d / 2}}{\Gamma(d / 2)}, \quad \widehat{\mathrm{d} \vartheta}=\omega_{d}^{-1} \mathrm{~d} \vartheta, \quad \int_{\mathbb{S}^{d-1}} \widehat{\mathrm{d} \vartheta}=1 .
$$

With these notations,

$$
\mu(r) \mathrm{d} r \mathrm{~d} \vartheta=\frac{v_{m}(x)}{1+|x|^{2}} \mathrm{~d} x=v_{m}^{2-m} \mathrm{~d} x \quad \text { and } \quad \nu(r) \mathrm{d} r \mathrm{~d} \vartheta=v_{m} \mathrm{~d} x .
$$

We define a directional average of a function $f$ by

$$
\widetilde{f_{\mu}}(\vartheta):=\int_{0}^{+\infty} f(r, \vartheta) \hat{\mu}(r) \mathrm{d} r \quad \text { with } \quad \hat{\mu}(r):=\frac{\mu(r)}{\int_{0}^{+\infty} \mu(s) \mathrm{d} s}
$$

and the global average of $f$ by

$$
\widetilde{f}:=\frac{\int_{\mathbb{R}^{d}} f v_{m}^{2-m} \mathrm{~d} x}{\int_{\mathbb{R}^{d}} v_{m}^{2-m} \mathrm{~d} x}=\iint_{(0, \infty) \times \mathbb{S}^{d-1}} f(r, \vartheta) \hat{\mu}(r) \mathrm{d} r \widehat{\mathrm{d} \vartheta}=\int_{\mathbb{S}^{d-1}} \widetilde{f_{\mu}}(\vartheta) \widehat{\mathrm{d} \vartheta} .
$$

In the case $d=1$, Theorem 2 of [4], also see [38], says that Inequality (A.1) holds with

$$
\mathcal{C}_{m, 1} \leq \frac{2 m}{1-m} \sup _{r>0}\left[\int_{r}^{+\infty} \mu(r) \mathrm{d} r \int_{0}^{r} \frac{\mathrm{d} r}{\nu(r)}\right]=\mathrm{K}(0)
$$

in which case we also have the estimate $\mathcal{C}_{m, 1} \leq \mathrm{K}(0) \leq 4 \mathcal{C}_{m, 1}$.

In case of radial functions, Inequality (A.1) takes the form:

$$
\int_{0}^{+\infty}\left|f(r)^{2}-\tilde{f}\right|^{2} \mu(r) \mathrm{d} r \leq \frac{1-m}{2 m} \mathcal{C}_{m, d}^{\mathrm{rad}} \int_{0}^{+\infty}\left|f^{\prime}(r)\right|^{2} \nu(r) \mathrm{d} r
$$

with

$$
\mathcal{C}_{m, d}^{\mathrm{rad}} \leq \frac{2 m}{1-m} \max \left\{\sup _{r>\eta}\left[\int_{r}^{+\infty} \mu(r) \mathrm{d} r \int_{\eta}^{r} \frac{\mathrm{d} r}{\nu(r)}\right], \sup _{r<\eta}\left[\int_{0}^{r} \mu(r) \mathrm{d} r \int_{r}^{\eta} \frac{\mathrm{d} r}{\nu(r)}\right]\right\}=\mathrm{K}(\eta) .
$$

It is straightforward to show that $\mathrm{K}(\eta)$ is finite, with the present choices of $\mu$ and $\nu$, for $m \in$ $\left(m_{*},(d-2) /(d-1)\right)$, and as above, $\mathcal{C}_{m, d}^{\mathrm{rad}} \leq \mathrm{K}(\eta) \leq 4 \mathcal{C}_{m, d}^{\mathrm{rad}}$.

We now focus on the case of non radial functions, with $d \geq 2$, and rewrite the left hand side of (A.1) in spherical coordinates.

$$
\begin{aligned}
\int_{\mathbb{R}^{d}}|f(x)-\widetilde{f}|^{2} v_{m}^{2-m} \mathrm{~d} x & =\omega_{d} \iint_{(0, \infty) \times \mathbb{S}^{d-1}}|f(r, \vartheta)-\widetilde{f}|^{2} \mu(r) \mathrm{d} r \widehat{\mathrm{d} \vartheta} \\
& =\omega_{d} \iint_{(0, \infty) \times \mathbb{S}^{d-1}}\left|f(r)-\widetilde{f_{\mu}}(\vartheta)+\widetilde{f_{\mu}}(\vartheta)-\widetilde{f}\right|^{2} \mu(r) \mathrm{d} r \widehat{\mathrm{d} \vartheta} \\
& \leq 2 \omega_{d}[(\mathrm{I})+(\mathrm{II})]
\end{aligned}
$$

with

$$
\begin{aligned}
& (\mathrm{I})=\iint_{(0, \infty) \times \mathbb{S}^{d-1}} \underset{\mid f}{f}(r, \vartheta)-\left.\widetilde{f_{\mu}}(\vartheta)\right|^{2} \mu(r) \mathrm{d} r \widehat{\mathrm{d} \vartheta}, \\
& (\mathrm{II})=\left.\iint_{(0, \infty) \times \mathbb{S}^{d-1}} \underset{f_{\mu}}{\widetilde{f}}\right|^{2} \mu(r) \mathrm{d} r \widehat{\mathrm{d} \vartheta}=\int_{\mathbb{S}^{d-1}}\left|\widetilde{f_{\mu}}-\widetilde{f}\right|^{2} \widehat{\mathrm{d} \vartheta} .
\end{aligned}
$$

We estimate (I) by (A.4) and get

$$
(\mathrm{I})=\iint_{(0, \infty) \times \mathbb{S}^{d-1}}\left|f(r)^{2}-\widetilde{f_{\mu}}(\vartheta)\right|^{2} \mu(r) \mathrm{d} r \widehat{\mathrm{d} \vartheta} \leq \frac{1-m}{2 m} \mathcal{C}_{m, d}^{\mathrm{rad}} \iint_{(0, \infty) \times \mathbb{S}^{d-1}}\left|\partial_{r} f(r, \vartheta)\right|^{2} \nu(r) \mathrm{d} r \widehat{\mathrm{d} \vartheta} .
$$


To estimate (II), we rely on the Poincaré inequality on the unit sphere $\mathbb{S}^{d-1}$,

$$
\int_{\mathbb{S}^{d-1}}|u-\hat{u}|^{2} \widehat{\mathrm{d} \vartheta} \leq \frac{1}{d-1} \int_{\mathbb{S}^{d-1}}\left|\nabla_{\vartheta} u\right|^{2} \widehat{\mathrm{d} \vartheta} \quad \forall u \in H^{1}\left(\mathbb{S}^{d-1}\right) .
$$

Here $\hat{u}:=\int_{\mathbb{S}^{d-1}} u \widehat{\mathrm{d} \vartheta}$. In the inequality, $1 /(d-1)$ is the optimal constant, as can be checked using spherical harmonic functions. See for instance [5, 9, 43]. The inequality itself can be recovered by various methods. For example, using the inverse stereographic projection, see [37], the optimal Sobolev inequality on $\mathbb{R}^{d}$ becomes

$$
\left(\int_{\mathbb{S}^{d-1}}|v|^{p} \widehat{\mathrm{d} \vartheta}\right)^{2 / p} \leq \int_{\mathbb{S}^{d-1}}|v|^{2} \widehat{\mathrm{d} \vartheta}+\frac{p-2}{d-1} \int_{\mathbb{S}^{d-1}}\left|\nabla_{\vartheta} v\right|^{2} \widehat{\mathrm{d} \vartheta},
$$

for any $u \in H^{1}\left(\mathbb{S}^{d-1}\right)$, with $p=2 d /(d-2), d \geq 3$. The inequality also holds true for any $p \in(2,2 d /(d-2))$ if $d \geq 3$ and for any $p>2$ if $d=2$, see [6]. Hence we recover the Poincaré inequality on $\mathbb{S}^{d-1}$ by writing $v=1+\varepsilon u$ and keeping only the terms of order $\varepsilon^{2}$ as $\varepsilon \rightarrow 0$.

We apply the Poincaré inequality with $u=\widetilde{f_{\mu}}$.

$$
\int_{\mathbb{S}^{d-1}}\left|\widetilde{f_{\mu}}-\widetilde{f}\right|^{2} \widehat{\mathrm{d} \vartheta} \leq \frac{1}{d-1} \int_{\mathbb{S}^{d-1}}\left|\nabla_{\vartheta} \widetilde{f_{\mu}}\right|^{2} \widehat{\mathrm{d} \vartheta}
$$

Recall that $|\nabla f|^{2}=\left|\partial_{r} f(r, \vartheta)\right|^{2}+\frac{1}{r^{2}}\left|\nabla_{\vartheta} f(r, \vartheta)\right|^{2}$. Using the Cauchy-Schwarz inequality and the estimate $r^{2} \mu(r) \mathrm{d} r \leq \nu(r) \mathrm{d} r$, we get

$$
\begin{aligned}
\int_{\mathbb{S}^{d-1}}\left|\nabla_{\vartheta} \widetilde{f_{\mu}}\right|^{2} \widehat{\mathrm{d} \vartheta} & \leq \iint_{(0, \infty) \times \mathbb{S}^{d-1}}\left|\nabla_{\vartheta} f(r, \vartheta)\right|^{2} \mu(r) \mathrm{d} r \widehat{\mathrm{d} \vartheta} \\
& \leq \iint_{(0, \infty) \times \mathbb{S}^{d-1}} \frac{1}{r^{2}}\left|\nabla_{\vartheta} f(r, \vartheta)\right|^{2} \nu(r) \mathrm{d} r \widehat{\mathrm{d} \vartheta}
\end{aligned}
$$

This proves that

$$
\text { (II) } \leq \frac{1}{d-1} \iint_{(0, \infty) \times \mathbb{S}^{d-1}} \frac{1}{r^{2}}\left|\nabla_{\vartheta} f(r, \vartheta)\right|^{2} \nu(r) \mathrm{d} r \widehat{\mathrm{d} \vartheta} .
$$

Summarizing, we have shown that

$$
\int_{\mathbb{R}^{d}}|f(x)-\tilde{f}|^{2} v_{m}^{2-m} \mathrm{~d} x \leq 2 \max \left\{\frac{1-m}{2 m} \mathcal{C}_{m, d}^{\mathrm{rad}}, \frac{1}{d-1}\right\} \int_{\mathbb{R}^{d}}|\nabla f|^{2} v_{m} \mathrm{~d} x .
$$

By undoing the change of coordinates as in (A.3), we get

$$
\int_{\mathbb{R}^{d}}|f(x)-\bar{f}|^{2} \mathrm{~d} \mu \leq \max \left\{2 \mathcal{C}_{m, d}^{\mathrm{rad}}, \frac{4 m}{(1-m)(d-1)}\right\} \int_{\mathbb{R}^{d}}|\nabla f|^{2} \mathrm{~d} \nu
$$

The bounds on $\mathrm{K}(\eta)$ follow by quite long but straightforward calculations, omitted here.

\section{Appendix B: Extension to exponents $m \leq 0$}

The presence at several instances of factors of the form $1 / m$ in the previous calculations may suggest that there is an essential divergence as $m \rightarrow 0$. In the present section we want to dispel that impression by introducing a normalization that is often used in the literature, consisting in a rescaling of the time variable of the form $\tau^{\prime}=m \tau$ that modifies equation (1.1) into

$$
\partial_{\tau^{\prime}} u=\nabla \cdot\left(u^{m-1} \nabla u\right) .
$$

One of the first consequences is that the new equation, that we will call modified fast diffusion equation for clarity, makes perfect sense as a nonlinear parabolic equation of singular type for all the range of exponents $m \in \mathbb{R}$ (including $m=0$ ), in particular for all $m<1$ that form the extended range of the fast diffusion. Such approach has been consistently used in [47] where it is shown that the effect on the self-similar solutions of Barenblatt type is just to eliminate the denominator $m$ in the formulas (1.2), (1.5). Note the rescaling to obtain (B.1) from the standard 
fast diffusion equation (1.1) when $m>0$ can also be done by changing the space variable in the form $x=\sqrt{m} x^{\prime}$ and not changing time.

Since the general theory (existence, uniqueness, estimates, special solutions and extinction) has been developed to the measure we need it, we can follow the different stages of the present paper with due attention to chasing the $m$ factors, and the results stated in Section 1 remain valid. For instance, the formula defining $\mathcal{F}[w]$ in Section 2.5 has to be replaced by

$$
\mathcal{F}[w]=-\int_{\mathbb{R}^{d}}[\log w-(w-1)] \mathrm{d} x .
$$

In the linearization of Section 4 there is no $m$ factor in the definition of operator $A_{m}$ and neither in the definition of $\mathrm{I}[g]$. Let us mention two other points of interest: the exponent $m_{*}$ becomes negative for $d=1,2,3$ and zero for $d=4$, but it still plays the same role of an important critical exponent separating different behavior types. On the other hand, the constant $\lambda_{m, d}$ that gives the decay rate in our main result has a finite positive value as $m \rightarrow 0$, according to Theorem A.1. This constant determines the rates in all results concerning the asymptotic behavior of the solutions and is not affected by our $m$-rescaling.

We have refrained from treating the extension to $m \leq 0$ in the main body of the paper in order to avoid further distractions in an already very technical matter. Whole details will appear separately.

ACKnowledgments. This project has been supported by the IFO project of the French Research Agency (ANR). A.B. acknowledges the support of a bourse Lavoisier. M.B. thanks CNRS, CEREMADE, Dpto. di Matematica of Politecnico di Torino and the Dpto. de Matemáticas of Universidad Autónoma de Madrid for post-doctoral grants during which this project has been carried out. J.L.V. was partially supported by Spanish Project MTM2005-08760-C02-01. A.B. and J.L.V. were partially supported by the ESF Programme "Global and geometric aspects of nonlinear partial differential equations".

(c) 2007 by the authors. This paper may be reproduced, in its entirety, for non-commercial purposes.

\section{REFERENCES}

[1] T. Aubin, Problèmes isopérimétriques et espaces de Sobolev, J. Differential Geometry, 11 (1976), pp. 573-598.

[2] D. BAKRY AND M. Émery, Diffusions hypercontractives, in Séminaire de probabilités, XIX, 1983/84, vol. 1123 of Lecture Notes in Math., Springer, Berlin, 1985, pp. 177-206.

[3] F. Barthe and C. Roberto, Sobolev inequalities for probability measures on the real line, Studia Math., 159 (2003).

[4] F. BArthe And C. Roberto, Modified logarithmic Sobolev inequalities on $\mathbb{R}, 2006$.

[5] W. Beckner, Sobolev inequalities, the Poisson semigroup, and analysis on the sphere $S^{n}$, Proc. Nat. Acad. Sci. U.S.A., 89 (1992), pp. 4816-4819.

[6] _ Sharp Sobolev inequalities on the sphere and the Moser-Trudinger inequality, Ann. of Math. (2), 138 (1993), pp. 213-242.

[7] A. Blanchet, M. Bonforte, J. Dolbeault, G. Grillo, and J.-L. Vázquez, Hardy-Poincaré inequalities and applications to nonlinear diffusions, C. R. Math. Acad. Sci. Paris, (2007).

[8] S. G. Bobkov And F. GöTze, Exponential integrability and transportation cost related to logarithmic Sobolev inequalities, J. Funct. Anal., 163 (1999), pp. 1-28.

[9] L. BočEK, Eine Verschärfung der Poincaré-Ungleichung, Časopis Pěst. Mat., 108 (1983), pp. 78-81.

[10] M. Bonforte And J. L. VÁzQuez, Fine asymptotics near extinction and elliptic Harnack inequalities for the fast diffusion equation. Preprint, April 2006.

[11] - Global positivity estimates and Harnack inequalities for the fast diffusion equation, J. Funct. Anal., 240 (2006), pp. 399-428.

[12] L. Caffarelli, R. Kohn, and L. Nirenberg, First order interpolation inequalities with weights, Compositio Math., 53 (1984), pp. 259-275.

[13] J. A. Carrillo, A. Jüngel, P. A. Markowich, G. Toscani, and A. Unterreiter, Entropy dissipation methods for degenerate parabolic problems and generalized Sobolev inequalities, Monatsh. Math., 133 (2001), pp. 1-82. 
[14] J. A. Carrillo, C. Lederman, P. A. Markowich, and G. Toscani, Poincaré inequalities for linearizations of very fast diffusion equations, Nonlinearity, 15 (2002), pp. 565-580.

[15] J. A. Carrillo And G. Toscani, Asymptotic $L^{1}$-decay of solutions of the porous medium equation to selfsimilarity, Indiana Univ. Math. J., 49 (2000), pp. 113-142.

[16] J. A. Carrillo And J. L. VÁzquez, Fine asymptotics for fast diffusion equations, Comm. Partial Differential Equations, 28 (2003), pp. 1023-1056.

[17] F. CATRINA AND Z.-Q. WANG, On the Caffarelli-Kohn-Nirenberg inequalities: sharp constants, existence (and nonexistence), and symmetry of extremal functions, Comm. Pure Appl. Math., 54 (2001), pp. 229-258.

[18] Y. Z. Chen And E. DiBenedetto, Hölder estimates of solutions of singular parabolic equations with measurable coefficients, Arch. Rational Mech. Anal., 118 (1992), pp. 257-271.

[19] S.-K. ChuA And R. L. Wheeden, Sharp conditions for weighted 1-dimensional Poincaré inequalities, Indiana Univ. Math. J., 49 (2000), pp. 143-175.

[20] P. Daskalopoulos And M. Del Pino, On the Cauchy problem for $u_{t}=\Delta \log u$ in higher dimensions, Math. Ann., 313 (1999), pp. 189-206.

[21] P. Daskalopoulos and N. Sesum, Eternal solutions to the Ricci flow on $\mathbb{R}^{2}$, Int. Math. Res. Not., (2006), pp. Art. ID 83610, 20.

[22] P. Daskalopoulos And N. Sesum, On the extinction profile of solutions to fast-diffusion. Preprint, 2006.

[23] E. B. DaviEs, Heat kernels and spectral theory, vol. 92 of Cambridge Tracts in Mathematics, Cambridge University Press, Cambridge, 1989.

[24] E. B. Davies And A. M. Hinz, Explicit constants for Rellich inequalities in $L_{p}(\Omega)$, Math. Z., 227 (1998), pp. 511-523.

[25] M. Del Pino and J. Dolbeault, Best constants for Gagliardo-Nirenberg inequalities and applications to nonlinear diffusions, J. Math. Pures Appl. (9), 81 (2002), pp. 847-875.

[26] M. Del Pino And M. SÁEz, On the extinction profile for solutions of $u_{t}=\Delta u^{(N-2) /(N+2)}$, Indiana Univ. Math. J., 50 (2001), pp. 611-628.

[27] J. Denzler and R. J. MCCann, Fast diffusion to self-similarity: complete spectrum, long-time asymptotics, and numerology, Arch. Ration. Mech. Anal., 175 (2005), pp. 301-342.

[28] A. Friedman And S. Kamin, The asymptotic behavior of gas in an n-dimensional porous medium, Trans. Amer. Math. Soc., 262 (1980), pp. 551-563.

[29] L. Gross, Logarithmic Sobolev inequalities, Amer. J. Math., 97 (1975), pp. 1061-1083.

[30] M. A. Herrero And M. Pierre, The Cauchy problem for $u_{t}=\Delta u^{m}$ when $0<m<1$, Trans. Amer. Math. Soc., 291 (1985), pp. 145-158.

[31] S.-Y. Hsu, Classification of radially symmetric self-similar solutions of $u_{t}=\Delta \log u$ in higher dimensions, Differential Integral Equations, 18 (2005), pp. 1175-1192.

[32] — Extinction profile of solutions of a singular diffusion equation, Commun. Appl. Anal., 9 (2005), pp. 6793.

[33] _ L Large time behavior of solutions of a singular diffusion equation in $\mathbb{R}^{n}$, Nonlinear Anal., 62 (2005), pp. 195-206.

[34] J. KInG, Self-similar behavior for the equation of fast nonlinear diffusion., Philos. Trans. R. Soc. Lond., Ser. A, 343 (1993), pp. 337-375.

[35] O. A. Ladyženskaja, V. A. Solonnikov, and N. N. Ural'Ceva, Linear and quasilinear equations of parabolic type, Translated from the Russian by S. Smith. Translations of Mathematical Monographs, Vol. 23, American Mathematical Society, Providence, R.I., 1967.

[36] C. Lederman And P. A. Markowich, On fast-diffusion equations with infinite equilibrium entropy and finite equilibrium mass, Comm. Partial Differential Equations, 28 (2003), pp. 301-332.

[37] E. H. LiEB, Sharp constants in the Hardy-Littlewood-Sobolev and related inequalities, Ann. of Math. (2), 118 (1983), pp. 349-374.

[38] L. Miclo, Quand est-ce que les bornes de Hardy permettent de calculer une constante de Poincaré exacte sur la droite? Preprint, 2006.

[39] L. Nirenberg, On elliptic partial differential equations, Ann. Scuola Norm. Sup. Pisa (3), 13 (1959), pp. 115162.

[40] F. Отто, The geometry of dissipative evolution equations: the porous medium equation, Comm. Partial Differential Equations, 26 (2001), pp. 101-174.

[41] M. Peletier And H. Zhang, Self-similar solutions of a fast diffusion equation that do not conserve mass., Differ. Integral Equ., 8 (1995), pp. 2045-2064.

[42] A. Rodriguez, J. L. Vazquez, and J. R. Esteban, The maximal solution of the logarithmic fast diffusion equation in two space dimensions, Adv. Differential Equations, 2 (1997), pp. 867-894.

[43] L. Saloff-Coste, Precise estimates on the rate at which certain diffusions tend to equilibrium, Math. Z., 217 (1994), pp. 641-677.

[44] G. Talenti, Best constant in Sobolev inequality, Ann. Mat. Pura Appl. (4), 110 (1976), pp. 353-372. 
[45] J. L. VÁzquez, Asymptotic behavior for the porous medium equation posed in the whole space, J. Evol. Equ., 3 (2003), pp. 67-118. Dedicated to Philippe Bénilan.

[46] — The Porous Medium Equation.Mathematical Theory, Oxford Mathematical Monographs. The Clarendon Press, Oxford University Press, Oxford, 2007.

[47] — Smoothing and decay estimates for nonlinear diffusion equations, vol. 33 of Oxford Lecture Notes in Maths. and its Applications, Oxford Univ. Press, 2006.

[48] J. L. VÁzquez, J. R. Esteban, And A. Rodríguez, The fast diffusion equation with logarithmic nonlinearity and the evolution of conformal metrics in the plane, Adv. Differential Equations, 1 (1996), pp. 21-50.

A. Blanchet: CRM, Apartat 50, 08193 Bellaterra, Spain. E-mail: blanchet@ceremade.dauphine.fr

M. Bonforte: Departamento de Matemáticas, Univ. Autónoma de Madrid, Campus de Cantoblanco, 28049 Madrid, Spain \& Dip. di Matematica, Politecnico di Torino, Corso Duca degli Abruzzi 24, 10129 Torino, Italy. E-mail: bonforte@ceremade.dauphine.fr

J. Dolbeault: CEREMADE, Univ. Paris Dauphine, Pl. de Lattre de Tassigny, 75775 Paris 16, France. E-mail: dolbeault@ceremade.dauphine.fr

G. Grillo: Dip. di Matematica, Politecnico di Torino, Corso Duca degli Abruzzi 24, 10129 Torino, Italy. E-mail: gabriele.grillo@polito.it

J.L. VÁzquez: Departamento de Matemáticas, Univ. Autónoma de Madrid, Campus de Cantoblanco, 28049 Madrid, Spain. E-mail: juanluis.vazquez@uam.es 